\title{
Combined Maximality Principles up to Large Cardinals
}

\author{
Gunter Fuchs \\ Institut für Mathematische Logik und Grundlagenforschung \\ Westfälische Wilhelms-Universität Münster \\ Einsteinstraße 62, 48149 Münster, Germany
}

March 31, 2008

\begin{abstract}
The motivation for this paper is the following: In [Fuc08] I showed that it is inconsistent with ZFC that the maximality principle for closed forcings holds at unboundedly many regular cardinals $\kappa$ (even only allowing $\kappa$ itself as a parameter in the maximality principle for $<\kappa$-closed forcings each time). So the question is whether it is consistent to have this principle at unboundedly many regular cardinals or at every regular cardinal below some large cardinal $\kappa$ (instead of $\infty$ ), and if so, how strong it is. It turns out that it is consistent in many cases, but the consistency strength is quite high. As a by-product, assuming the consistency of a supercompact cardinal, I show that it is consistent that the least weakly compact cardinal is indestructible.
\end{abstract}

\section{Introduction}

Let $\varphi(a)$ be a formula about a parameter $a$, and let $\Gamma$ be a class of notions of forcing (like c.c.c. forcings, $\sigma$-closed forcings, proper forcings, etc.), defined by a formula $\psi_{\Gamma}$ which may use parameters. Then $\varphi(a)$ is $\Gamma$-forceable if there is a notion of forcing $\mathbb{P}$ in $\Gamma$ such that $\mathbb{P} \Vdash \varphi(\check{a})$. It is $\Gamma$-necessary if for every $\mathbb{P} \in \Gamma, \mathbb{P} \Vdash \varphi(\check{a})$. It is $\Gamma$-forceably necessary if there is a forcing $\mathbb{P}$ such that $\mathbb{P}$ forces that $\varphi(\check{a})$ is necessary. More precisely, this means that $\mathbb{P} \Vdash \forall \mathbb{Q} \quad\left(\psi_{\Gamma}(\mathbb{Q}) \Longrightarrow \mathbb{Q} \Vdash \varphi(\check{a})\right)$.

The Maximality Principle for forcings in $\Gamma$ with parameters in $P$, abbreviated as $\mathrm{MP}_{\Gamma}(P)$ is the scheme of formulae expressing that every formula with parameters in $P$ that is $\Gamma$-forceably necessary is true. It is a very natural axiom scheme that was first introduced by Stavi and Väänänen in [SV01], where they focused on 
the case where $\Gamma$ is the collection of all c.c.c. forcings. It was then rediscovered and investigated in a more general setting by Hamkins in [Ham03]. The most general and abstract analysis of Maximality Principles is probably George Leibman's dissertation [Lei04]. In [Fuc08], I looked at the case where $\Gamma$ is one of the following, for a fixed regular cardinal $\kappa$ :

- The class of all $<\kappa$-closed forcing notions,

- the class of all $<\kappa$-directed-closed forcing notions,

- the class $\operatorname{Col}(\kappa)$, which consists of all forcings of the form $\operatorname{Col}(\kappa, \lambda)$ or $\operatorname{Col}(\kappa,<\lambda)$, where $\lambda$ ranges over all ordinals.

What got me interested in these classes was that they have an intriguing number of consequences which can be found in the aforementioned paper. A basic fact about the relationship between these principles, for fixed $\kappa$, is that

$$
\mathrm{MP}_{\mathrm{Col}(\kappa)}(P) \Longrightarrow \mathrm{MP}_{<\kappa \text {-directed-closed }}(P) \Longrightarrow \mathrm{MP}_{<\kappa \text {-closed }}(P) .
$$

Only parameter sets $P \subseteq H_{\kappa^{+}}$can consistently be allowed. In this paper, $P=H_{\kappa^{+}}$ is the default, while the resulting principles are usually referred to as the boldface closed maximality principles. A remark on smaller parameter sets:

$$
\operatorname{MP}_{\Gamma}(\{\kappa\}) \Longleftrightarrow \operatorname{MP}_{\Gamma}\left(H_{\kappa} \cup\{\kappa\}\right),
$$

where $\Gamma$ is one of the $<\kappa$-closed classes of forcing. I refer to these principles as the lightface closed maximality principles at $\kappa$.

The aspect of the research on these principles that I am focussing on in the present paper has to do with the possibility of combining these principles, in the sense that they may hold at several regular cardinals at the same time.

Definition 1.1. Let $T$ be a constant symbol. Let $\bigwedge_{\tau \in T} \mathrm{MP}_{<\tau-\text { dir. cl. }}\left(H_{\tau^{+}}\right)$denote the scheme consisting of the sentences expressing:

$\forall \bar{\kappa} \in T \quad(\bar{\kappa}$ is regular $\Longrightarrow$

$$
\left.\forall x \in H_{\bar{\kappa}^{+}}(\varphi(x) \text { is }<\bar{\kappa} \text {-directed-closed-forceably necessary } \Longrightarrow \varphi(x))\right),{ }^{1}
$$

for every formula $\varphi(x)$ with free variable $x$. I shall also express this scheme by saying that the directed closed Maximality Principle holds on T. If $\kappa$ is a regular cardinal and $T$ is the set of regular cardinals which are less than $\kappa$, then I'll express $\bigwedge_{\tau \in T} \mathrm{MP}_{<\tau-\text { dir. cl. }}\left(H_{\tau^{+}}\right)$by saying that the directed closed Maximality Principle holds below $\kappa$, and if $T$ consists of the regular cardinals less than or equal to $\kappa$, then I'll say that the directed closed Maximality Principle holds up to $\kappa$.

\footnotetext{
${ }^{1}$ Formally, $T$ occurs as a constant symbol in the scheme. The interpretation of this symbol will be clear, so I shall omit the distinction between the symbol and its interpretation.
} 
The following theorem was proved in [Fuc08]:

Theorem 1.2. Assuming $\mathrm{ZFC}_{A}+\operatorname{otp}(A)=\alpha<\infty+" A$ is discrete and $A$ consists of regular fully reflecting cardinals", ${ }^{2}$ there is a forcing extension which satisfies

$$
\text { ZFC }+\bigwedge_{\begin{array}{c}
\gamma \text { among the first } \\
\alpha \text { regular cardinals }
\end{array}} \mathrm{MP}_{<\gamma-\text { dir. cl. }}\left(H_{\gamma^{+}}\right) .
$$

The reason why I am focussing on the Maximality Principles for closed and directed closed forcing is the following lemma from [Fuc08], which essentially shows that the Maximality Principle for collapses cannot be combined:

Lemma 1.3. If $\mathrm{MP}_{\mathrm{Col}\left(\kappa_{0}\right)}\left(H_{\kappa_{0}} \cup\left\{\kappa_{0}\right\}\right)$ holds, then there can be no $\kappa_{1}>\kappa_{0}^{+}$such that $\mathrm{MP}_{<\kappa_{1}-\text { closed }}\left(H_{\kappa_{1}+}\right)$ holds.

The argument shows also, for example, that there can be no $\kappa_{1}>\kappa_{0}^{++}$such that $\mathrm{MP}_{<\kappa_{0}-\text { closed }}\left(H_{\kappa_{0}} \cup\left\{\kappa_{0}\right\}\right)$ and $\mathrm{MP}_{<\kappa_{1}-\text { closed }}\left(H_{\kappa_{1}} \cup\left\{\kappa_{1}\right\}\right)$ hold simultaneously.

Surprisingly there are general limitations to the extent to which the closed Maximality Principles can be combined:

Theorem 1.4 ([Fuc08], Thm. 6.9). The theory ZFC $+\bigwedge_{\gamma \in \operatorname{Reg}} \mathrm{MP}_{<\gamma-\text { closed }}\left(H_{\gamma} \cup\{\gamma\}\right)$ is inconsistent. ${ }^{3}$

The argument also shows that the lightface closed Maximality Principles cannot hold on an unbounded class of regular cardinals. The situation is somehow a little bit like in the case of DC and $\alpha-\mathrm{AC}$ : For any $\alpha$, there is an inner model of a forcing extension of the universe where the $\alpha-\mathrm{AC}$ holds but DC fails, but if the $\alpha-\mathrm{AC}$ holds for every $\alpha$, then this implies DC (see [Jen66]). So the question I am investigating in the present work is whether the Maximality Principles can be combined below or up to large cardinals of various strengths, or if they can hold on unbounded or otherwise large sets below some large cardinal. So naively, the question is whether $\infty$ can be replaced with a regular $\kappa$ which satisfies strong axioms of infinity.

In section 2, I am building models where the directed closed Maximality Principles hold below a large cardinal. I also explain why the consistency strength of certain combinations is quite high: If the closed Maximality Principles hold on a measure one set below a measurable cardinal, then the set of indestructibly weakly

\footnotetext{
${ }^{2}$ Here, $A$ is a predicate symbol which is added to the language of set theory. $\mathrm{ZFC}_{A}$ is the usual axiom scheme, where replacement and separation is now demanded for every formula in the enriched language. A discrete class of ordinals is one which is disjoint from the collection of its limit points.

${ }^{3} \mathrm{Reg}$ is the class of all regular cardinals here.
} 
compact cardinals below that cardinal has measure one. I elaborate on the strength of indestructible weak compactness some more. Section 3, deals with the construction of models where the directed closed Maximality Principles hold up to a large cardinal, and section 3.2, takes up the thread on indestructible weak compactness, building models where the least weakly compact cardinal is indestructible. ${ }^{4}$ This is somewhat surprising, since indestructibly weakly compact cardinals have certain properties reminiscent of supercompactness - but this result shows that they have almost no reflection properties.

Finally, in section 3.3, I consider combinations of the Maximality Principles up to rather large cardinals like partially supercompact ones, or Woodinized supercompact cardinals.

\section{Closed Maximality Principles below a large cardinal}

In this section, I am interested in the following way of combining closed maximality principles:

Definition 2.1. I shall say that a cardinal $\rho$ is fully reflecting if the scheme $\mathrm{V}_{\rho} \prec \mathrm{V}$ holds. Let $R$ be a set of regular fully reflecting cardinals and $T$ a set of regular cardinals. For $\tau \in T$, let $\rho(\tau) \simeq \min (R \backslash(\tau+1))$ be the least member of $R$ above $\tau$, if it exists. If $\rho: T \rightarrow R$ is a bijection, then I shall say that the pair $\langle T, R\rangle$ is interweaved. In that case, let $\operatorname{Col}(T,<R)$ be the reverse Easton iteration of collapses $\operatorname{Col}(\tau,<\rho(\tau))$.

Using this terminology, Theorem 5.3 of [Fuc08] can be restated as follows:

Theorem 2.2. Suppose that $\langle T, R\rangle$ is an interweaved pair. Then forcing with $\operatorname{Col}(T,<R)$ produces models of $\bigwedge_{\tau \in T} \mathrm{MP}_{<\tau-\text { dir. cl. }}\left(H_{\tau^{+}}\right)$.

Let $R^{\prime}$ be a set of regular fully reflecting cardinals. Then there is a canonical interweaved pair $\langle T, R\rangle$ given by $R^{\prime}$ : $R$ consists of the regular members of $R^{\prime}$ which are no limits of $R^{\prime}$, and in order to define the canonical $T$, I define the function $\rho^{-1}$ with domain $R$ as follows. Given $\xi \in R$, let $\bar{\xi}=\sup (R \cap \xi)$. Then

$$
\rho^{-1}(\xi)= \begin{cases}\bar{\xi} & \text { if } \bar{\xi} \text { is a regular infinite cardinal } \\ \bar{\xi}^{+} & \text {otherwise. }\end{cases}
$$

\footnotetext{
${ }^{4}$ When I say that a weakly compact cardinal $\kappa$ is indestructible, I always mean that it is indestructible by $<\kappa$-closed forcing. So it stays weakly compact in any forcing extension obtained by $<\kappa$-closed forcing.
} 
Finally, let $T=\operatorname{ran}\left(\rho^{-1}\right)$. Then obviously, the function to $\rho$ witnesses that $\langle T, R\rangle$ is an interweaved pair. I refer to $\operatorname{Col}(T,<R)$ as the canonical collapse induced by $R^{\prime}$. If $\kappa$ is a regular fully reflecting cardinal and $R^{\prime}$ is the set of regular fully reflecting cardinals less than $\kappa$, then I will refer to the canonical collapse induced by $R^{\prime}$ as the canonical collapse below $\kappa$. If $R^{\prime}$ is the set of regular fully reflecting cardinals less than or equal to $\kappa$, then the canonical collapse induced by $R^{\prime}$ is the canonical collapse up to $\kappa$.

Lemma 2.3. Let $\kappa$ be a limit of regular fully reflecting cardinals. ${ }^{5}$ If $G$ is generic for the canonical collapse below $\kappa$, then in $\mathrm{V}[G]$, the boldface Maximality Principle for directed closed forcings holds below $\kappa$. If, in addition, $\kappa$ is regular (and hence inaccessible), then $\kappa$ remains inaccessible in $\mathrm{V}[G] .^{6}$

Proof. It is an immediate consequence of Theorem 2.2 that the postulated Maximality Principles hold - note that the $\mathrm{V}[G]$-regular cardinals below $\kappa$ are precisely the members of $T$. The canonical collapse below $\rho$ was explicitly designed to achieve this. $\kappa$ 's inaccessibility is preserved since the canonical collapse below $\kappa$ satisfies the $\kappa$-c.c. - it is a reverse Easton iteration.

I shall try to work my way up through the large cardinal hierarchy in the following.

Lemma 2.4. Let $\kappa$ be a fully reflecting Mahlo cardinal. Then in forcing extensions obtained by the canonical collapse below $\kappa, \kappa$ is still Mahlo and the Maximality Principle for directed closed forcings holds below $\kappa$.

Proof. Note that since $\kappa$ is a fully reflecting Mahlo cardinal, it is a limit of fully reflecting regular cardinals. So let $\mathbb{P}=\operatorname{Col}(T,<R)$ be the canonical collapse below $\kappa$, let $G$ be $\mathrm{V}$-generic for $\mathbb{P}$, let $p \in G$ be a condition in $\mathbb{P}$, and let $\dot{C}$ be a name that is forced by $p$ to be a club subset of $\kappa$. Let's assume that $\dot{C}$ is a nice name for a subset of $\check{\kappa}$. I have to find a member of $\dot{C}^{G}$ that's regular, from $\mathrm{V}[G]$ 's point of view.

Consider the structure $M=\left\langle\mathrm{V}_{\kappa}, \in, \dot{C}, \Vdash_{\mathbb{P}},\{p\}\right\rangle$. Since $\kappa$ is inaccessible, $M$ is

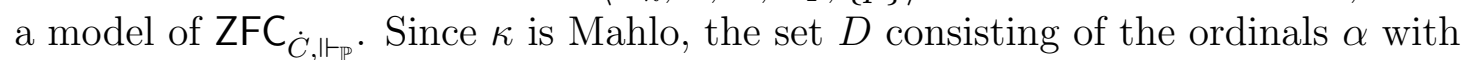
$M \mid \mathrm{V}_{\alpha} \prec M$ is club in $\kappa^{7}$. So since $\kappa$ is Mahlo, there is some regular $\bar{\kappa} \in D$. Let

\footnotetext{
${ }^{5}$ The "statement" that $\kappa$ is a limit of fully reflecting regular cardinals can be expressed as a scheme. Namely, it should be viewed as an abbreviation of the statement that the set of regular $\bar{\kappa}<\kappa$ such that $\mathrm{V}_{\bar{\kappa}} \prec \mathrm{V}_{\kappa}$ is unbounded in $\kappa$, together with the scheme expressing that $\kappa$ is fully reflecting.

${ }^{6}$ As was pointed out earlier, a fully reflecting Mahlo cardinal implies this. It is more than needed, though: The set of regular fully reflecting cardinals below a fully reflecting Mahlo cardinal which are limits of regular fully reflecting cardinals is stationary in the Mahlo cardinal.

${ }^{7}$ If $N$ is a model and $X$ is a subset of the domain of $N$, then I denote by $N \mid X$ the reduct of $N$ to $X$.
} 
$\bar{M}=M \mid \mathrm{V}_{\bar{\kappa}}$. Necessarily, $\overline{\mathbb{P}}:=\mathbb{P} \cap \mathrm{V}_{\bar{\kappa}}=\operatorname{Col}(T \cap \bar{\kappa},<(R \cap \bar{\kappa}))$. Since $\bar{M} \prec M$, it follows that $\bar{\kappa}$ is a limit of regular fully reflecting cardinals, and that $\mathbb{P} \cap \mathrm{V}_{\bar{\kappa}}$ is the canonical collapse below $\bar{\kappa}$. Let's split $\operatorname{Col}(T,<R)$ as

$$
\operatorname{Col}(T,<R) \cong \operatorname{Col}(T \cap \bar{\kappa},<(R \cap \bar{\kappa})) * \operatorname{Col}(T \cap[\bar{\kappa}, \kappa),<(R \cap(\bar{\kappa}, \kappa))) .
$$

Let $G=G \uparrow \bar{\kappa} * G \uparrow[\bar{\kappa}, \kappa)$ be split accordingly. By elementarity, $p$ forces via $\overline{\mathbb{P}}$ over $\bar{M}$ that $\dot{C} \cap \mathrm{V}_{\bar{\kappa}}$ is a club class. Note that $\overline{\mathbb{P}}$ is a class forcing from $\bar{M}$ 's point of view. But it is an iteration with increasing closure, so the forcing theorem holds in $\bar{M}$ for $\bar{P}$. So $\dot{C}^{G \uparrow \bar{\kappa}}=\dot{C}^{G} \cap \bar{\kappa}$ is club in $\bar{\kappa}$. So $\bar{\kappa} \in \dot{C}^{G}$. By Lemma $2.3, \bar{\kappa}$ is inaccessible in $\mathrm{V}[G \uparrow \bar{\kappa}]$, and since the tail forcing is $<\bar{\kappa}$-closed over $\mathrm{V}[G \uparrow \bar{\kappa}], \bar{\kappa}$ remains inaccessible in $\mathrm{V}[G]=\mathrm{V}[G \uparrow \bar{\kappa}][G \uparrow[\bar{\kappa}, \kappa)]$.

Let ZFC* be a finite fragment of ZFC which includes the powerset axiom and the axiom of choice and which is sufficiently strong. I shall need the following lemmas in several lifting arguments to follow.

Lemma 2.5. Let $\mathbb{P}$ be a forcing iteration of length $\kappa$, where $\kappa$ is regular, $\mathbb{P}$ has size $\kappa$, and $\mathbb{P}=\mathbb{P}_{\kappa}$ is formed as a limit with bounded supports. Also, assume that for any $\alpha<\kappa$, there is a $\beta<\kappa$ such that it is forced via $\mathbb{P}_{\beta}$ that $\overrightarrow{\dot{Q}} \uparrow[\beta, \kappa)$ is $<\alpha$-closed, and that for every $\gamma<\kappa, \overline{\overline{\mathbb{P}_{\gamma}}}<\kappa$.

Let $M$ be a transitive model of ZFC* (whether it is set-sized or a proper class does not matter), let ${ }^{<\kappa} M \subseteq M$, and suppose that $\mathbb{P} \in M$. Then if $G$ is $\mathbb{P}$-generic over $\mathrm{V}$,

$$
\mathrm{V}[G] \cap{ }^{<\kappa} M[G] \subseteq M[G] .
$$

We say that $M[G]$ is $<\kappa$-closed in $\mathrm{V}[G]$ to express this. Also, if ${ }^{\kappa} M \subseteq M$, then $M[G]$ is $\kappa$-closed in $\mathrm{V}[G]$ :

$$
\mathrm{V}[G] \cap{ }^{\kappa} M[G] \subseteq M[G] .
$$

Proof. Let's concentrate on $<\kappa$-closedness first. Let $\theta=$ On $\cap M$, where $\theta=$ On is possible. I shall first prove that $\mathrm{V}[G] \cap{ }^{<\kappa} \theta \subseteq M[G]$.

So let $\alpha<\kappa$, and $\left\langle x_{\nu} \mid \nu<\alpha\right\rangle \in \mathrm{V}[G]$, with $x_{\nu}<\theta$, for every $\nu<\alpha$. By the assumptions on $\mathbb{P}$, there is some $\gamma$ s.t. $\vec{x} \in \mathrm{V}[G \uparrow \gamma]$. Let $\dot{f}$ be a $\mathbb{P}_{\gamma}$-name for $\vec{x}$, and pick a condition $p_{0} \in G \uparrow \gamma$ which forces wrt. $\mathbb{P}_{\gamma}$ that $\dot{f}$ is an $\alpha$-sequence of ordinals less than $\theta$. Now, for $\nu<\alpha$, let $A_{\nu}$ be the set of conditions below $p_{0}$ (in $\mathbb{P}_{\gamma}$ ) which decide the value of $\dot{f}(\nu)$. Every $A_{\nu}$ is dense below $p_{0}$ and has size at most $\overline{\overline{\mathbb{P}_{\gamma}}}$, which is less than $\kappa$. So $A:=\bigcup_{\nu<\alpha}\{\nu\} \times A_{\nu}$ has size less than $\kappa$ as well, by the regularity of $\kappa$. Now let $g: A \longrightarrow \theta$ be such that $p \Vdash_{\mathbb{P}_{\gamma}} \dot{f}(\nu)=g(\nu, p)$. Clearly, $g \in M$, as $M$ is $<\kappa$-closed. But $\vec{x}$ is definable from $g$ and $G$, namely, $x_{\nu}=\xi$, where $g(\nu, p)=\xi$, for some $p \in G$.

Now let $\vec{y}=\left\langle y_{\nu} \mid \nu<\beta\right\rangle \in \mathrm{V}[G] \cap{ }^{<\kappa} M[G]$. Since $M$ is a model of ZFC*, $M[G]$ still satisfies a strong enough fragment of ZFC to conclude that $M[G]=$ 
$\bigcup_{\alpha<\theta} \mathrm{V}_{\alpha}^{M[G]}$. So it makes sense to define the sequence $\vec{r}=\left\langle r_{\nu} \mid \nu<\beta\right\rangle$ of ordinals below $\theta$ by letting $r_{\nu}$ be the least $\gamma$ with $y_{\nu} \in \mathrm{V}_{\gamma}^{M[G]}$. So $\vec{r} \in M[G]$, by what was shown in the first part. So $\rho:=\sup \vec{r} \in M[G]$, since $\vec{r} \in \mathrm{V}_{\gamma}^{M[G]}$, for some $\gamma<\theta$ (so that $\sup \vec{r}<\gamma$ ). Now by the axiom of choice in $M[G]$, let $f: \lambda \longrightarrow \mathrm{V}_{\rho}^{M[G]}$ be a bijection with $f \in M[G]$. Let $\bar{y}_{\nu}=f^{-1}\left(y_{\nu}\right)$, for $\nu<\beta$. Then again by the first part of the proof, $\left\langle\bar{y}_{\nu} \mid \nu<\beta\right\rangle \in M[G]$. Hence, so is $\vec{y}$.

The proof showing that $\kappa$-closedness is preserved is simpler and hence omitted. It works for arbitrary posets $\mathbb{P} \in M$ which have cardinality at most $\kappa$.

The following Lemma is a crucial ingredient in most lifting arguments. It is well-known, but I state it with proof due to its central role for my purposes.

Lemma 2.6. Let $j: M \longrightarrow N$ be an elementary embedding, where $M$ and $N$ are transitive models of ZFC. Let $\mathbb{P} \in M$ be a notion of forcing. Let $G$ be $\mathbb{P}$-generic over $M$, and let $H$ be $j(\mathbb{P})$-generic over $N$ such that $j$ " $G \subseteq H$. Then there is an elementary embedding $j^{\prime}: \mathrm{V}[G] \longrightarrow M[H]$ which extends $j$.

Proof. Define $j^{\prime}\left(\tau^{G}\right):=j(\tau)^{H}$. Both the correctness of this definition and the elementarity of $j^{\prime}$ can be seen as follows: If $M[G] \models \varphi(\tau)$, then there is a $p \in G$ with $M \models\left(p \Vdash_{\mathbb{P}} \varphi(\tau)\right)$. Then $N \models\left(j(p) \Vdash_{j(\mathbb{P})} \varphi(j(\tau))\right.$, and since $j(p) \in H$, this means that $N[H] \models \varphi\left(j(\tau)^{G}\right)$.

Lemma 2.7. Let $\kappa$ be weakly compact and fully reflecting. If $G$ is $\mathrm{V}$-generic for the canonical collapse below $\kappa$, then in $\mathrm{V}[G], \kappa$ is still weakly compact, and the boldface Maximality Principle for directed closed forcings holds below $\kappa$.

Proof. Let $\mathbb{P}=\operatorname{Col}(T,<R)$, where $\langle T, R\rangle$ is the canonical interweaved pair given by the fact that $\kappa$ is a regular limit of fully reflecting regular cardinals.

I shall use the fact that a cardinal $\tau$ is weakly compact iff for every transitive model $P \ni \tau$ of size $\tau$, there is another transitive model $P^{\prime}$ and an elementary embedding $\pi: P \longrightarrow P^{\prime}$ with critical point $\tau$.

So let $\tilde{M} \in \mathrm{V}[G]$ be transitive, have $\mathrm{V}[G]$-cardinality $\kappa$ and have $\kappa \in \tilde{M}$. I shall also use the following fact:

(1) $H_{\kappa^{+}}^{\mathrm{V}[G]}=H_{\kappa^{+}}[G]$.

Proof. For the substantial direction, if $x \in H_{\kappa^{+}}^{\mathrm{V}[G]}$, then $x$ is coded by a subset $a$ of $\kappa$. Let $\tau$ be a nice name for a subset of $\kappa$ with $a=\tau^{G}$. Then clearly, $\tau \in H_{\kappa^{+}}$, and so, $a \in H_{\kappa^{+}}[G]$. Now $x$ can be decoded from $a$ in $H_{\kappa^{+}}[G]$, since this is a ZFC ${ }^{-}$-model.

So we can pick $\tau \in H_{\kappa^{+}}$s.t. $\tau^{G}=\tilde{M}$. Let $\bar{M} \in \mathrm{V}$ have the following properties:

1. $\bar{M}$ is a transitive model of $\mathrm{ZF}^{-}$, 
2. $\bar{M} \in H_{\kappa^{+}}$,

3. $\mathrm{TC}(\{\tau\}) \subseteq \bar{M}$

4. $\mathbb{P} \in \bar{M}$,

5. ${ }^{<\kappa} \bar{M} \subseteq \bar{M}$

6. $\bar{M} \models \mathrm{ZFC}^{*}$.

The existence of such an $\bar{M}$ is guaranteed by the inaccessibility of $\kappa$. In particular, $\tilde{M} \in M:=\bar{M}[G]$.

Let $j: \bar{M} \longrightarrow \bar{N}$ be a weakly compact embedding, w.l.o.g. given by an ultrafilter $U$ on $\bar{M}$ (external to $\bar{M}$ ). I shall produce a weakly compact embedding from $M$ to a generic extension of $N:=\bar{N}[G]$ in $\mathrm{V}[G]$.

(2) $\bar{M}$ is closed under $<\kappa$-sequences in $\mathrm{V}$.

Proof. Since $\bar{M}$ is closed under $<\kappa$-sequences, so is $\bar{N}$. This is a general fact about ultrapowers: If $\vec{x}=\left\langle x_{\nu} \mid \nu<\alpha\right\rangle \in{ }^{<\kappa} \bar{N}$, then every $x_{\nu}$ has the form $j\left(f_{\nu}\right)(\kappa)$, for some $f_{\nu} \in \bar{M}$. So the whole sequence $\left\langle f_{\nu}\right| \nu\langle\alpha\rangle$ is a member of $\bar{M}$. I am now looking for a function $g$ which represents $\vec{x}$ in the ultrapower. Using the Loś theorem, $g$ has this property if for every $\nu<\alpha$,

$$
\left\{\mu<\kappa \mid M \models\left(g(\mu) \text { is a sequence of length } \alpha \text { and } g(\mu)_{\nu}=f_{\nu}(\mu)\right)\right\} \in U .
$$

So defining $g(\mu)=\left\langle f_{\nu}(\mu) \mid \nu<\alpha\right\rangle$ for $\mu<\kappa$ yields a function with the desired property.

(3) $\mathrm{V}[G] \cap\left({ }^{<\kappa} M\right) \subseteq M$ and $\mathrm{V}[G] \cap\left({ }^{<\kappa} N\right) \subseteq N$

Proof. This is Lemma 2.5.

Set $\mathbb{Q}=j(\overrightarrow{\mathbb{Q}}) \uparrow[\kappa, j(\kappa))^{G}$.

(4) $\mathbb{P}=(j(\mathbb{P}))_{\kappa}$, and $N \models \mathbb{Q}$ is $<\kappa$-closed.

Proof. By elementarity of $j$.

(5) $\mathrm{V}[G] \models \mathbb{Q}$ is $<\kappa$-closed. 
Proof. This follows from the previous two points.

This last point can be made use of in order to find a filter $H$ in $\mathrm{V}[G]$ which is $\mathbb{Q}$-generic over $\bar{N}[G]$. One just has to construct a decreasing $\kappa$-chain of conditions $\vec{p}$ in $\mathbb{Q}$ such that $p_{\alpha} \in D_{\alpha}$, where $\vec{D}$ enumerates (in $\mathrm{V}[G]$ ) the dense subsets of $\mathbb{Q}$ which are in $N$. Since $N$ has size $\kappa$ in $\mathrm{V}[G]$ and $\mathbb{Q}$ is $<\kappa$-closed, this can be done in $\mathrm{V}[G]$.

Finally, the embedding $j: \bar{M} \longrightarrow \bar{N}$ can now be lifted to an embedding $j^{\prime}: M \longrightarrow N[H]$, by Lemma 2.6. This is possible because $j$ " $G \subseteq G * H$, as $\mathbb{P}$ is a direct limit.

So $j^{\prime}$ is a weakly compact embedding for $M$ in $\mathrm{V}[G]$. We have to get one for $\tilde{M}$. But note that since $\tilde{M} \in M$, it follows that $j^{\prime}\left\lceil\tilde{M}: \tilde{M} \longrightarrow_{\Sigma_{\omega}} j^{\prime}(\tilde{M})\right.$, and $j^{\prime}(\tilde{M})$ is transitive: $\tilde{M}$ is transitive, $\tilde{M} \in M$, and $M$ is transitive, so $M$ thinks that $\tilde{M}$ is transitive, so $N[H]$ thinks that $j(\tilde{M})$ is transitive. Moreover, $N[H]$ is transitive, so that $j(\tilde{M})$ is indeed transitive. And it's obvious that the critical point of $j^{\prime} \uparrow \tilde{M}$ is $\kappa$, so we've found the desired weakly compact embedding of $\tilde{M}$.

Note that up to now, the constructions were very economic in the following sense. If $\varphi(\kappa)$ expresses one of the following large cardinal concepts: " $\kappa$ is inaccessible", " $\kappa$ is Mahlo", " $\kappa$ is weakly compact", then I constructed models in which $\varphi(\kappa)$ is true and the boldface Maximality Principles for directed closed forcings hold below $\kappa$, starting from a model in which $\varphi(\kappa)+" \kappa$ is a limit of fully reflecting regular cardinals" holds. ${ }^{8}$ This will (provably) not be possible for the stronger large cardinal concepts I am going to analyze later. But let me state that in the realm of small large cardinals, it is possible to go the other way:

Lemma 2.8. Let $\varphi(\kappa)$ express one of the following large cardinal concepts: " $\kappa$ is inaccessible", " $\kappa$ is Mahlo", " $\kappa$ is weakly compact". Then the following theories are transitive model equiconsistent, locally in $\kappa:^{9}$

1. $\mathrm{ZFC}+\bigwedge_{\tau \in \operatorname{Reg} \cap \kappa} \mathrm{MP}_{<\tau-\text { dir. cl. }}\left(H_{\tau^{+}}\right)+\varphi(\kappa)$,

2. ZFC $+" \kappa$ is a limit of fully reflecting regular cardinals" $+\varphi(\kappa)$.

Proof. The direction from 2 to 1 has been shown in the previous sequence of lemmas. For the converse, it has been shown in Theorem 3.8 of [Fuc08] that $\mathrm{MP}_{<\tau-\text { closed }}\left(H_{\tau^{+}}\right)$(which is weaker than $\mathrm{MP}_{<\tau-\text { dir. cl. }}\left(H_{\tau^{+}}\right)$) implies that $\tau^{+}$is inaccessible in $L$ and that $L_{\tau^{+}} \prec L{ }^{10}$ So if the boldface Maximality Principles for directed closed forcings hold below a limit cardinal $\kappa$, then the set of $L$-cardinals

\footnotetext{
${ }^{8}$ In case $\kappa$ is Mahlo, $\kappa$ is a fully reflecting limit of fully reflecting regular cardinals if and only if $\kappa$ is fully reflecting.

${ }^{9}$ For a formal definition of the concept of local transitive model equiconsistency, I refer the reader to [Fuc08].

${ }^{10}$ It has also been shown in [Fuc08] that $\mathrm{MP}_{<\tau-\text { dir. cl. }}\left(H_{\tau^{+}}\right)$implies $\mathrm{MP}_{<\tau-\text { closed }}\left(H_{\tau^{+}}\right)$.
} 
which are fully reflecting regular cardinals in $L$ is unbounded in $\kappa$. So since the class of fully reflecting cardinals is closed, it follows that $L_{\kappa} \prec L$, too. Finally, the large cardinal concepts considered go down from $\mathrm{V}$ to $L$, so that the theory mentioned in 2 holds in $L$.

The following remark explains a posteriori why I am mainly interested in combinations of the boldface closed maximality principles.

Remark 2.9. The following theories are equiconsistent:

1. ZFC $+\kappa$ is Mahlo $+\bigwedge_{\tau \in \operatorname{Reg} \cap \kappa} \mathrm{MP}_{<\tau-\text { dir. cl. }}\left(H_{\tau^{+}}\right)$,

2. ZFC $+\kappa$ is Mahlo $+\bigwedge_{\tau \in \operatorname{Reg} \cap \kappa} \mathrm{MP}_{<\tau-\text { dir. cl. }}\left(H_{\tau} \cup\{\tau\}\right)$.

Proof. For the substantial direction, starting from a model of the second theory, we get that in $L, \kappa$ is a Mahlo limit of fully reflecting ordinals, so $\kappa$ is a fully reflecting Mahlo cardinal in $L$. So $\kappa$ is a Mahlo limit of regular fully reflecting cardinals in $L$, and hence we can force the first theory over $L$, using the canonical collapse below $\kappa$.

Lemma 2.10. Let $\kappa$ be measurable, as witnessed by some normal ultrafilter $U$ on $\kappa$, and fully reflecting. Let $\langle T, R\rangle$ be an interweaved pair such that $R \subseteq \kappa$ and $T \notin U$. Let $j: \mathrm{V} \longrightarrow_{U} M$ be the ultrapower embedding. Then in a generic extension of $\mathrm{V}$ by $j\left(\mathrm{Col}(T,<R), \kappa\right.$ is measurable and $\bigwedge_{\tau \in T} \mathrm{MP}_{<\tau-\text { dir. cl. }}\left(H_{\tau^{+}}\right)$holds.

Proof. This is a variant of Kunen-Paris forcing. If $T$ is bounded in $\kappa$, then $\mathbb{P}:=$ $\operatorname{Col}(T,<R)$ is small forcing and hence preserves the measurability of $\kappa$, so the interesting case is that $T$ is unbounded in $\kappa$, and so $\mathbb{P}$ is an iteration of length $\kappa$. Let $\bar{G}$ be $\mathbb{P}$-generic over V.

Let $j: \mathrm{V} \longrightarrow M$ be the ultrapower embedding given by $U$. So $M$ is $\kappa$-closed in $\mathrm{V}$, and hence, $M[\bar{G}]$ is $\kappa$-closed in $\mathrm{V}[\bar{G}]$, by Lemma 2.5. Note that the tail forcing $\mathbb{P}^{\prime}=j(\overrightarrow{\mathbb{Q}}) \uparrow[\kappa, j(\kappa))^{\bar{G}}$ is $<\kappa^{+}$-closed in $M[\bar{G}]$, and hence also in $\mathrm{V}[\bar{G}]$.

Let $G^{\prime}$ be $j(\overrightarrow{\mathbb{Q}}) \uparrow[\kappa, j(\kappa))^{G \kappa}$-generic over $\mathrm{V}[\bar{G}]$, and set $G=\bar{G} * G^{\prime}$. Note that $\mathbb{P}=j(\mathbb{P})_{\kappa}$. We know that the desired maximality principle holds in $\mathrm{V}[\bar{G}]$. By [Fuc08, Lemma 4.2], they still hold in $\mathrm{V}[\bar{G}]\left[G^{\prime}\right]$. So we are left to show that $\kappa$ is measurable there.

By the Kunen-Paris argument (see [Jec03, Thm. 21.3]), the embedding $j$ : $\mathrm{V} \longrightarrow M$ now can be lifted to an embedding $j^{\prime}: \mathrm{V}[G \uparrow \kappa] \longrightarrow M[G]$, simply by setting $j\left(\tau^{G \uparrow \kappa}\right)=j(\tau)^{G}$. The point is that $j^{\text {" }}(G\lceil\kappa) \subseteq G$, because for any $p \in \mathbb{P}$, $j(p) \uparrow[\kappa, j(\kappa)) \equiv 1$, as the support of $p$ is bounded in $\kappa$, so that the support of $j(p)$ is the same as the support of $p$.

This embedding can be defined in $\mathrm{V}[G]$. Let $U^{\prime}$ be the normal ultrafilter on $\mathcal{P}(\kappa)^{\mathrm{V}[G\lceil\kappa]}$ derived from $j^{\prime}$. Note that $M[G \uparrow \kappa]$ believes that $j(\overrightarrow{\mathbb{Q}}) \uparrow[\kappa, j(\kappa))^{G \uparrow \kappa}$ is $<\kappa^{+}$-closed. This is because $\kappa \notin j(T)$, as $T$ has measure 0 . Also, ${ }^{\kappa} M \subseteq M$ and 
$\mathbb{P}$ has size $\kappa$, so that Lemma 2.5 yields that $\mathrm{V}\left[G\lceil\kappa] \cap\left({ }^{\kappa} M[G\lceil\kappa]) \subseteq M[G \uparrow \kappa]\right.\right.$. This means that $\mathrm{V}[G \uparrow \kappa]$ also believes that $j(\overrightarrow{\mathbb{Q}}) \uparrow[\kappa, j(\kappa))^{G \uparrow \kappa}$ is $<\kappa^{+}$-closed. So $\mathcal{P}(\kappa) \cap \mathrm{V}[G \uparrow \kappa]=\mathcal{P}(\kappa) \cap \mathrm{V}[G]$. This means that $U^{\prime}$ is a normal ultrafilter on $\kappa$ from the point of view of $\mathrm{V}[G]$ as well, so we're done.

Note: If $2^{\kappa}=\kappa^{+}$, then just forcing with $\operatorname{Col}(T,<R)$ will produce the desired model. For if $\bar{G}$ is generic for that forcing, we can find a filter $G^{\prime}$ which is generic for the tail forcing over $M[\bar{G}]$ already in $\mathrm{V}[\bar{G}]$. The tail forcing has cardinality $j(\kappa)$ in $M[\bar{G}]$, so we may view every subset of it as a subset of $j(\kappa)$. Every subset of $j(\kappa)$ in $M[G]$ has the form $\tau^{\bar{G}}$, for some nice $\mathbb{P}$-name $\tau \in M$ for a subset of $j(\kappa)$. Since $\mathbb{P}$ has size $\kappa$ in $M$, every such name can be viewed as a function from $j(\kappa)$ to $\mathcal{P}(\kappa)$ which is a member of $M$. So every such function has the form $[f]_{U}$, for some function $f: \kappa \longrightarrow \mathrm{V}, f \in \mathrm{V}$, such that for every $\alpha<\kappa, f(\alpha): \kappa \longrightarrow \mathcal{P}(\alpha)$ (by Łóz's theorem). There are $2^{\kappa}=\kappa^{+}$such functions. So $\mathrm{V}[\bar{G}]$, the collection of dense subsets of the tail forcing which exist in $M[\bar{G}]$ has cardinality $\kappa^{+}$. The tail forcing is $\leq \kappa$-closed, so one can construct in $\mathrm{V}[\bar{G}]$. a filter $G^{\prime}$ which is generic over $M[G]$ for the tail forcing. Then we can lift $j$ to $j^{\prime}: \mathrm{V}[\bar{G}] \longrightarrow M[\bar{G}]\left[G^{\prime}\right]$, showing that $\kappa$ is measurable in $\mathrm{V}[\bar{G}]$.

The assumptions made in the previous lemma were not too strong:

Lemma 2.11. If the theory expressing that the closed maximality principle holds on an unbounded subset of a measurable cardinal is consistent, then so is the theory expressing that there is a measurable fully reflecting cardinal.

Proof. Work in a universe where the first theory holds. If there is an inner model with a Woodin cardinal, then the second theory is easily seen to be true in a segment of that inner model: If $W$ is the inner model and $\delta$ is Woodin in $W$, then the set of measurable cardinals below $\delta$ in $W$ is stationary in $\delta$ (from the point of view of $W$ ). So by considering the club of $\alpha<\delta$ such that $\mathrm{V}_{\alpha}^{W}$ is closed under Skolem functions for $\mathrm{V}_{\delta}^{W}$, we get a member $\kappa$ of the intersection of these sets. So $\kappa$ is measurable in $\mathrm{V}_{\delta}^{W}$ and $\mathrm{V}_{\kappa}^{W} \prec \mathrm{V}_{\delta}^{W}$. So $\mathrm{V}_{\delta}^{W}$ is a model of the desired theory.

If there is no inner model with a Woodin cardinal, then the core model $K$ exists, by a recent result due to Jensen and Steel. ${ }^{11}$ Assume that $\kappa$ is measurable and the closed maximality principle holds on an unbounded subset $T$ of $\kappa$. It follows from [Fuc08] that $K \mid \tau^{+} \prec K$, for any $\tau \in T$, because $K$ is forcing invariant. So since the set of $\tau^{+}, \tau \in T$, in unbounded in $\kappa$, it follows that $K \mid \kappa \prec K$. And $\kappa$ is measurable in $K$, so $K$ is a model of the desired theory.

Dropping the requirement that the set on which the closed Maximality Principle holds has measure 0 increases the consistency strength drastically:

\footnotetext{
${ }^{11}$ It is no longer necessary to work below a measurable cardinal $\Omega$, as in [Ste96]. The results showing how to eliminate this technical assumption are still unpublished.
} 
Remark 2.12. If $\kappa$ is measurable, $U$ is a normal ultrafilter on $\kappa$ and the set of $\bar{\kappa}<\kappa$ such that $\mathrm{MP}_{<\bar{\kappa} \text {-closed }}\left(H_{\bar{\kappa}} \cup\{\bar{\kappa}\}\right)$ holds has $U$-measure 1 , then the set of indestructibly weakly compact cardinals below $\kappa$ has $U$-measure $1 .^{12}$

Proof. $\mathrm{MP}_{<\kappa-\text { dir. cl. }}\left(H_{\kappa} \cup\{\kappa\}\right)$ holds in $M:=\mathrm{Ult}(\mathrm{V}, U)$ by Łóz's theorem: Fixing a formula $\varphi(\vec{x})$, the statement "for every $\vec{a} \in H_{\alpha} \cup\{\alpha\}$, if $\varphi(\vec{a})$ is $<\alpha$-directed-closedforceably necessary, then $\varphi(\vec{a})$ " is true for $U$-measure 1 many $\alpha$. So by normality of $U$, it is true of $\kappa$ in $M$. Moreover, since $\mathrm{V}_{\kappa+1}^{M}=\mathrm{V}_{\kappa+1}$, it follows that $\kappa$ is weakly compact in $M$. Now Theorem 3.6 of [Fuc08] states that $\mathrm{MP}_{<\kappa \text {-closed }}\left(H_{\kappa} \cup\right.$ $\{\kappa\})$ already implies $<\kappa$-closed-generic $\Sigma_{2}^{1}\left(H_{\kappa}\right)$-absoluteness. The statement that $\kappa$ is weakly compact is a $\Pi_{2}^{1}\left(H_{\kappa}\right)$ statement, and consequently pertains to $<\kappa$ closed-generic extensions of $M$, so that $\kappa$ is indestructibly weakly compact in $M$. Applying Eóz's theorem again yields the claim.

The consistency strength of an indestructibly weakly compact cardinal is at least that of a non-domestic mouse ${ }^{13}$, so the assumption that the closed maximality principle holds on a measure one set below a measurable cardinal has at least that strength.

In order to formulate the assumption I need in order to get the directed closed Maximality Principle on a measure one set below a measurable cardinal, let me recall the following definition.

Definition 2.13 ([Men74]). Let $\mathcal{F}$ be a normal fine measure on $\mathcal{P}_{\kappa} \lambda$, and let $f: \kappa \longrightarrow \kappa$ be a function. Then $\sigma(\mathcal{F}, f)$ is the statement that

$$
\left\{x \in \mathcal{P}_{\kappa} \lambda \mid f(\operatorname{otp}(x \cap \kappa)) \geq \operatorname{otp}(x)\right\} \in \mathcal{F} .
$$

In terms of the ultrapower embedding $j: \mathrm{V} \longrightarrow \mathcal{F} M=\operatorname{Ult}(\mathrm{V}, \mathcal{F})$, this means that $j(f)(\kappa) \geq \lambda$. Say that $\sigma(\mathcal{F}, f)$ weakly fails if $j(f)(\kappa) \leq \lambda$.

While Menas was mainly interested in normal measures $\mathcal{F}$ for which there is a function such that $\sigma(\mathcal{F}, f)$ holds (this is what he refers to as $\sigma(\mathcal{F})$ ), I need a normal filter $\mathcal{F}$ which weakly fails to satisfy $\sigma(\mathcal{F}, f)$, for some specific function $f$. It was pointed out in [Men74, Prop. 2.29 and the following remark] that it is the failure of $\sigma$ that has high consistency strength, e.g., if there is a measure on $\mathcal{P}_{\kappa}(\lambda)$ which fails to satisfy $\sigma$, then there is a normal measure on $\kappa$ concentrating on supercompact cardinals. It is weaker than almost hugeness, though. And I only need the weak failure of $\sigma(\mathcal{F}, f)$, for some specific $f$, not simultaneously for all $f$.

\footnotetext{
${ }^{12} \mathrm{~A}$ cardinal $\tau$ is indestructibly weakly compact if $\tau$ is weakly compact in any forcing extension by a $<\tau$-closed forcing.

${ }^{13}$ See [Fuc08] and [JSSS07] for a further discussion. The strength of a non-domestic mouse exceeds what is known as the $A D_{\mathbb{R}}$ hypothesis. For more on indestructible weak compactness, the reader is referred to [AH01] and the forthcoming [Fuc].
} 
Remark 2.12 shows an interesting aspect of the next lemma, because it provides a new way of producing an indestructibly weakly compact cardinal, other than using the Laver preparation to make a supercompact cardinal indestructible. ${ }^{14}$

Lemma 2.14. Assume that $\kappa$ is fully reflecting. Let $f: \kappa \longrightarrow \kappa$ be the function which maps $\xi<\kappa$ to the least $\rho>\xi$ such that $\rho$ is regular and $\mathrm{V}_{\rho} \prec \mathrm{V}_{\kappa}$. Assume there is a normal measure $\mathcal{F}$ on $\mathcal{P}_{\kappa} \rho$ such that $\sigma(\mathcal{F}, f)$ weakly fails. Then there is a forcing $\mathbb{P}$ such that if $G$ is $\mathbb{P}$-generic over $\mathrm{V}$, then in $\mathrm{V}[G], \kappa$ is measurable, $\mathrm{MP}_{<\kappa-\text { dir. cl. }}\left(H_{\kappa^{+}}\right)$holds, and the set of $\lambda<\kappa$ which are regular and at which $\mathrm{MP}_{<\lambda \text {-dir. cl. }}\left(H_{\lambda^{+}}\right)$holds has measure 1 wrt. any normal ultrafilter on $\kappa$ in $\mathrm{V}[G]$.

Proof. Given a set $X$ of ordinals, let $X^{\prime}$ denote the set of limit points of $X$ which are less than the supremum of $X$, and let Reg be the class of regular cardinals. Let $A$ be the set of regular reflecting cardinals below $\kappa$, and set $T=(A \cap \kappa)^{\prime} \cap \operatorname{Reg}$. This is the intersection of a club set with a set that has measure one wrt. every normal ultrafilter on $\kappa$, hence the same is true of $T$. Note that every member of $T$ is strongly inaccessible. Let $j: \mathrm{V} \longrightarrow \mathcal{F} M=\mathrm{Ult}(\mathrm{V}, \mathcal{F})$, so $j(f)(\kappa) \leq \rho$, where $f$ and $\mathcal{F}$ are as in the statement of the lemma. Let $R$ be the image of $T$ under $f$. Then $\langle T, R\rangle$ is an interweaved pair, and $\overline{\mathbb{P}}=\operatorname{Col}(T,<R)$ will force the closed maximality principle at every $\delta \in T$.

By elementarity, $j(\overline{\mathbb{P}})=(\operatorname{Col}(j(T),<j(R)))^{M}$. So $j(\overline{\mathbb{P}})$ can be decomposed as

$$
j(\mathbb{P}) \cong \operatorname{Col}(T,<R)^{M} * \operatorname{Col}\left(T^{\prime},<R^{\prime}\right)^{M} .
$$

Note that $j(T)$ and $j(R)$ are defined from $j(A)$ in $M$ as $T$ and $R$ were defined from $A$ in $\mathrm{V}$. Actually, we get the following decomposition:

$$
j(\overline{\mathbb{P}}) \cong \operatorname{Col}(T,<R) * \dot{\operatorname{Col}}(\kappa,<j(f)(\kappa))^{M} * \dot{\operatorname{Col}}(\tilde{T},<\tilde{R})^{M} .
$$

Namely, since $\kappa$ is a regular limit of $j(A)$ in $M$, it follows that $\kappa \in j(T)$, which explains why the second component in the decomposition collapses to $\kappa$.

The least member of $\tilde{T}$ is an inaccessible cardinal (in $M$ ) greater than $j(f)(\kappa)$. Since $M$ is closed under $\rho$-sequences,

$$
\mathbb{P}:=\operatorname{Col}(T,<R)^{\mathrm{V}} * \dot{\operatorname{Col}}(\kappa,<j(f)(\kappa))^{\mathrm{V}}=\operatorname{Col}(T,<R)^{M} * \operatorname{Col}(\kappa,<j(f)(\kappa))^{M},
$$

so we get the decomposition

$$
j(\mathbb{P}) \cong \mathbb{P} * \underbrace{\operatorname{Col}(\tilde{T}, \tilde{R})^{M}}_{\dot{\mathbb{Q}}} .
$$

\footnotetext{
${ }^{14}$ The Laver preparation makes a supercompact cardinal indestructibly so under $<\kappa$-directedclosed forcing. It follows that $\kappa$ 's weak compactness will be indestructible under $<\kappa$-closed forcing; see [Fuc, Observation 2.2]
} 
Let $G$ be $\mathbb{P}$-generic over V. I will employ a master condition argument due to Silver, which I will give in detail, since it will be necessary to apply variations of it in more and more complicated contexts. Note that if $p \in \mathbb{P}$, then it is a sequence $p=\left\langle p_{\alpha} \mid \alpha \leq \kappa\right\rangle$, such that for any $\alpha \leq \kappa, p \uparrow \alpha \in \mathbb{P}_{\alpha}$ and $p\left\lceil\alpha \Vdash_{\mathbb{P}_{\alpha}} p_{\alpha} \in \dot{\mathbb{Q}}_{\alpha}\right.$.

Set:

$$
D=\left\{\tau^{G} \mid \exists p \in G \exists p^{\prime} \quad j(p)=\left\langle p^{\prime}, \tau\right\rangle \in \mathbb{P} * \dot{\mathbb{Q}}\right\} .
$$

(1) $D \in M[G]$, and $\overline{\bar{D}}^{M[G]}<\rho$.

Proof. The point is that $M[G]$ is $\rho$-closed in V $[G]$. In particular, $j \uparrow(G \uparrow(\kappa+1))$ as well as $G$ are in $M[G]$, and $D$ is definable from these parameters. Moreover, $\overline{\bar{D}}^{M[G]} \leq \overline{\bar{G}}^{M[G]}$, and that is $\left(j(f)(\kappa)^{<\kappa}\right)^{M[G]}$. But $j(f)(\kappa)=\left(\kappa^{+}\right)^{M[G]}$, and in $M[G],\left(\kappa^{+}\right)^{<\kappa}=\kappa^{+}$, since $2^{\kappa}=\kappa^{+}$there, as $j(f)(\kappa)$ is inaccessible in $M . \quad \square_{(1)}$

(2) $M[G] \models$ “Q் is $<\rho^{\prime}$-directed-closed", for some $M[G]$-inaccessible $\rho^{\prime}>j(f)(\kappa)$.

Proof. This is because in $M, \dot{\mathbb{Q}}$ is a $\mathbb{P}$-name for $\operatorname{Col}(\tilde{T}, \tilde{R})$, and $\min (\tilde{T})>j(f)(\kappa)$, as $T$ consisted only of regular limit points of $A$.

(3) D is a directed subset of $\dot{\mathbb{Q}}^{G}$.

Proof. Let $p, q \in D$. Let $\bar{p}, \bar{q} \in G$ be such that, letting $j(\bar{p})=\left\langle p^{\prime}, \dot{p}\right\rangle \in \mathbb{P} * \dot{\mathbb{Q}}$ and $j(\bar{q})=\left\langle q^{\prime}, \dot{q}\right\rangle \in \mathbb{P} * \dot{\mathbb{Q}}, p=\dot{p}^{G}$ and $q=\dot{q}^{G}$. Since $G$ is generic for $\mathbb{P}$, there is an $\bar{r} \leq_{\mathbb{P}} \bar{p}, \bar{q}$ with $\bar{r} \in G$. So

$(*) j(\bar{r}) \leq_{j(\overline{\mathbb{P}})} j(\bar{p}), j(\bar{q})$.

View $\bar{r}$ as a function with domain $\kappa+1$, such that $\bar{r} \uparrow \kappa \in \overline{\mathbb{P}}$. Then $j(\bar{r} \uparrow \kappa) \uparrow$ $(\kappa+1) \in G$ : To see this, note that $\bar{r} \uparrow \kappa$ is eventually trivial, because $\overline{\mathbb{P}}$ is a direct limit. So

$$
j(\bar{r} \uparrow \kappa) \uparrow \kappa=\bar{r} \uparrow \kappa \text { and } j(\bar{r} \uparrow \kappa) \uparrow[\kappa, j(\kappa)) \equiv 1 .
$$

So, $j(\bar{r}) \uparrow(\kappa+1) \geq \bar{r} \in G$, which shows that $j(\bar{r} \uparrow \kappa) \uparrow(\kappa+1) \in G$, as claimed.

Now view $j(\bar{r})$ as a pair $\left\langle r^{\prime}, \dot{r}\right\rangle \in \mathbb{P} * \dot{\mathbb{Q}}$. So $r^{\prime}=j(\bar{r}) \uparrow(\kappa+1) \in G$, which means that $r:=\dot{r}^{G} \in D$. By $(*)$,

$$
r^{\prime} \leq_{\mathbb{P}} p^{\prime}, q^{\prime} \text { and } r^{\prime} \Vdash_{\mathbb{P}} \dot{r} \leq_{\mathbb{Q}} \dot{p}, \dot{q} .
$$

So, since $r^{\prime} \in G, r \leq_{\mathbb{Q}} p, q$, which shows that $D$ is directed.

Now pick a master condition $a \in \mathbb{Q}$ below each condition in $D$. This is possible by (1), (2) and (3). Let $H$ be $\mathbb{Q}$-generic over $\mathrm{V}[G]$ with $a \in H$.

(4) $j$ " $G \subseteq G * H$. 
Proof of (4). Of course, one has to view $G * H$ as a subset of $j(\mathbb{P})$ in order to make sense of the inclusion at hand. Let $\bar{p} \in G$, and view its image as $j(\bar{p})=\left\langle p^{\prime}, \dot{p}\right\rangle \in$ $\mathbb{P} * \dot{\mathbb{Q}}$. Then $p:=\dot{p}^{G} \in D$, and so, $a \leq_{\mathbb{Q}} p$. So $p \in H$. Moreover, the argument in the proof of (3) showed that $p^{\prime} \geq \bar{p}$, so that $p^{\prime} \in G$. So $j(\bar{p})=\left\langle p^{\prime}, \dot{p}\right\rangle \in G * H$, as claimed.

So $j: \mathrm{V} \longrightarrow M$ lifts to an embedding $j^{\prime}: \mathrm{V}[G] \longrightarrow M[G][H]$, by Lemma 2.6. Finally, the normal ultrafilter $U$ derived from $j^{\prime}$ is in $\mathrm{V}[G]$ (so we're done), since we left sufficiently large gaps between the cardinals at which we forced the closed maximality principle. The point is that $\mathbb{Q}$ is $\leq j(f)(\kappa)$-closed not only in $M[G]$ but also in $\mathrm{V}[G]$, as $M[G]$ is $\rho$-closed in $\mathrm{V}[G], j(f)(\kappa) \leq \rho$ and $\mathbb{Q}$ is $<\rho^{\prime}$-closed in $M[G]$ and $\rho^{\prime}>j(f)(\kappa)$. So we can view $U$ as a $\left(2^{\kappa}\right)^{\mathrm{V}[G][H]}$-sequence of subsets of $\kappa$ each of which is in $\mathrm{V}[G]$. But $\left(2^{\kappa}\right)^{\mathrm{V}[G]}=j(f)(\kappa)$, as $j(f)(\kappa)$ is inaccessible (not only in $M$, but also in $\mathrm{V}$, because $M$ is $\rho$-closed and $\rho \leq j(f)(\kappa))$, so the forcing adding $G$ is $j(f)(\kappa)$-c.c. over $\mathrm{V}$. So $U$ is the image of a $j(f)(\kappa)$-sequence (which is in $\mathrm{V}[G][H])$ of members of $\mathcal{P}(\kappa)^{\mathrm{V}[G]}$, and hence it is in $\mathrm{V}[G]$, by the fact that $\mathbb{Q}$ is $\leq j(f)(\kappa)$-closed in $\mathrm{V}[G]$.

In Lemma 3.21, I construct a model where the closed Maximality Principle holds at every regular cardinal below a Woodinized supercompact cardinal.

\section{Closed Maximality Principles up to a large cardinal}

In this section I am aiming at producing models in which the closed maximality principle holds on an unbounded set of regular cardinals less than $\kappa$ and at $\kappa$ itself, where $\kappa$ satisfies some strong axiom of infinity.

I shall say that the closed maximality principle holds up to $\kappa$ if it holds at every regular cardinal less than or equal to $\kappa$.

\subsection{Up to small large cardinals}

Combining up to inaccessible or Mahlo cardinals works without any complications:

Lemma 3.1. Let $\varphi(\kappa)$ express either that $\kappa$ is inaccessible or that $\kappa$ is Mahlo. Then the following theories are transitive model equiconsistent, locally in $\kappa$ and $\rho$ :

1. $\mathrm{ZFC}+\bigwedge_{\tau \in \operatorname{Reg} \cap(\kappa+1)} \mathrm{MP}_{<\tau-\text { dir. cl. }}\left(H_{\tau^{+}}\right)+\varphi(\kappa)+\rho=\kappa^{+}$,

2. $\mathrm{ZFC}+\mathrm{V}_{\kappa} \prec \mathrm{V}+\varphi(\kappa)+\kappa<\rho+\rho$ is regular $+\mathrm{V}_{\rho} \prec \mathrm{V}$.

Proof. For the direction from 1 to 2, since the large cardinal properties involved go down to $L$, the constructible universe will model the second theory. And vice 
versa, forcing with the canonical collapse up to $\rho$ will produce a model of the first theory. Note that this collapse is the same as the canonical collapse up to $\kappa$, followed by one further collapse, which is $<\kappa$-closed and hence preserves the large cardinal property of $\kappa$.

Definition 3.2. Let $A$ be a set and $\lambda$ an ordinal. Let $j: \mathrm{V} \longrightarrow M$ be an elementary embedding, where $M$ is transitive. Say that $j$ moves $A$ correctly up to $\lambda$ if $j(A) \cap \mathrm{V}_{\lambda}=A \cap \mathrm{V}_{\lambda}$. A cardinal $\kappa$ is said to be $(A, \lambda)$-supercompact if there is a $\lambda$-supercompact embedding $j: \mathrm{V} \longrightarrow M$ with critical point $\kappa$ (i.e., $M$ is transitive, ${ }^{\lambda} M \subseteq M, j\lceil\kappa=\mathrm{id}, j(\kappa)>\kappa \text { and } j(\kappa) \geq \lambda)^{15}$ that moves $A$ correctly up to $\lambda$. $\kappa$ is $A$-supercompact up to $\lambda^{\prime}$ if it is $(A, \lambda)$-supercompact, for every $\lambda<\lambda^{\prime}$. Analogously, $\kappa$ is almost $(A, \lambda)$-huge if there is an almost huge embedding $j: \mathrm{V} \longrightarrow M$ with critical point $\kappa$ (so that ${ }^{<j(\kappa)} M \subseteq M$ and $j(\kappa) \geq \lambda$ ) which moves $A$ correctly up to $\lambda$. It is almost $A$-huge up to $\lambda^{\prime}$ if it is almost $(A, \lambda)$-huge, for every $\lambda<\lambda^{\prime}$.

If $\mathcal{F}$ is a fine normal measure on $\mathcal{P}_{\kappa} \lambda$, then I will write

$$
\pi: \mathrm{V} \longrightarrow \mathcal{F} M
$$

to express that $\pi$ is the canonical elementary embedding from $\mathrm{V}$ into the transitivized ultrapower of $\mathrm{V}$ by $\mathcal{F}$, which I also denote by $M=\operatorname{Ult}(\mathrm{V}, \mathcal{F})$.

I shall give an upper bound on the consistency strength of the assumption made in the next lemma after its proof.

Lemma 3.3. Assume that $\kappa<\rho$ are regular fully reflecting cardinals and $\kappa$ is $(A, \rho+1)$-supercompact, where $A$ consists of the regular fully reflecting cardinals that are less than or equal to $\rho$, where $\rho=\min (A \backslash(\kappa+1))$. Then there is a forcing $\mathbb{P}$ such that if $G$ is $\mathbb{P}$-generic over $\mathrm{V}$, then in $\mathrm{V}[G], \kappa$ is measurable, $\mathrm{MP}_{<\kappa-\text { dir. cl. }}\left(H_{\kappa^{+}}\right)$holds, and the set of $\lambda<\kappa$ which are regular and at which $\mathrm{MP}_{<\lambda \text {-dir. cl. }}\left(H_{\lambda^{+}}\right)$holds has measure 1 wrt. any normal ultrafilter on $\kappa$ in $\mathrm{V}[G]$.

Proof. The proof follows that of Lemma 2.14 very closely, which is why I will omit most of the details. As in that proof, let $T=(A \cap \kappa)^{\prime} \cap \operatorname{Reg}$. Let $j: \mathrm{V} \longrightarrow M$ be an embedding witnessing that $\kappa$ is $(A, \rho+1)$-supercompact. So $j(A) \cap(\rho+1)=A$ and ${ }^{\rho} M \subseteq M$.

Now, for $\tau \in T$, let $f(\tau)$ be the least member of $A$ greater than $\tau$. Let $R$ be the image of $T$ under $f$. Then $\langle T, R\rangle$ is an interweaved pair, and $\overline{\mathbb{P}}=\operatorname{Col}(T,<R)$ will force the maximality principle at every $\delta \in T \cup\{\kappa\}$. Let $\mathbb{P}=\overline{\mathbb{P}} * \dot{\operatorname{Col}}(\kappa,<\rho)$. So $\mathbb{P}$ forces the maximality principle at $\kappa$ in addition.

\footnotetext{
${ }^{15}$ It is well-known how to avoid the apparent metamathematical difficulties arising from the quantification over classes. E.g., in the case of supercompactness, it suffices to consider ultrapower embeddings induced by fine normal measures on some $\mathcal{P}_{\kappa}(\lambda)$.
} 
Note that $j(T)$ and $j(R)$ are defined from $j(A)$ in $M$ as $T$ and $R$ were defined from $A$ in $\mathrm{V}$. So since $A$ is moved correctly up to $\rho+1$, and since $T \in U$, it follows that $j(\overline{\mathbb{P}})$ decomposes as

$$
j(\mathbb{P}) \cong \mathbb{P} * \underbrace{\operatorname{Col}(\tilde{T}, \tilde{R})^{M}}_{\dot{\mathbb{Q}}} .
$$

Let $G$ be $\mathbb{P}$-generic over V. Set:

$$
D=\left\{\tau^{G} \mid \exists p \in G \exists p^{\prime} \quad j(p)=\left\langle p^{\prime}, \tau\right\rangle \in \mathbb{P} * \dot{\mathbb{Q}}\right\} .
$$

The following claims are proved as before:

(1) $D \in M[G]$, and $\overline{\bar{D}}^{M[G]}=\rho$.

(2) $M[G] \models$ "Q্Q is $<\rho^{\prime}$-directed-closed", for some $\rho^{\prime}>\rho$.

(3) $D$ is a directed subset of $\dot{\mathbb{Q}}^{G}$.

Now pick a master condition $a \in \mathbb{Q}$ below each condition in $D$, which can be done in $M[G]$, by (1), (2) and (3). Let $H$ be $\mathbb{Q}$-generic over $\mathrm{V}[G]$ with $a \in H$. Like before, it follows that

(4) $j$ “ $G \subseteq G * H$.

Now lift $j: \mathrm{V} \longrightarrow M$ to $j^{\prime}: \mathrm{V}[G] \longrightarrow M[G][H]$. The normal ultrafilter $U$ derived from $j^{\prime}$ is in $\mathrm{V}[G]$. For we can view $U$ as a $\left(2^{\kappa}\right)^{\mathrm{V}[G][H]}$-sequence of subsets of $\kappa$ each of which is in $\mathrm{V}[G]$. But $\left(2^{\kappa}\right)^{\mathrm{V}[G]}=\rho$, as $\rho$ is inaccessible in $\mathrm{V}$, so the forcing adding $G$ is $\rho$-c.c. So $U$ is the image of a $\rho$-sequence (which is in $\mathrm{V}[G][H]$ ) of members of $\mathcal{P}(\kappa)^{\mathrm{V}[G]}$, and hence it is in $\mathrm{V}[G]$, by the fact that the tail forcing adding $H$ is $\leq \rho$-closed in $\mathrm{V}[G]$.

Remark 3.4. If the theory ZFC + "there is an almost huge cardinal" is consistent, then so are the assumptions of the previous lemma.

Proof. Let $j: \mathrm{V} \longrightarrow M$ be an almost huge embedding with critical point $\kappa$. Let $A=\left\{\alpha \mid \alpha\right.$ is regular and $\left.\mathrm{V}_{\alpha} \prec \mathrm{V}_{\kappa}\right\}$. Let $\rho=\min (j(A) \backslash(\kappa+1))$. Let $\mathcal{F}$ be the normal measure on $\mathcal{P}_{\kappa}(\rho+1)$ derived from $j$. Then $\mathcal{F} \in M$, as $\rho<j(\kappa)$ and ${ }^{<j(\kappa)} M \subseteq M$. Let $j^{\prime}: M \longrightarrow \mathcal{F} N$ be the ultrapower by $\mathcal{F}$. Then $j^{\prime} \uparrow \rho+1=j \uparrow$ $\rho+1$. So in $M$, the statement that there is a regular limit point $\kappa^{\prime}$ of $j(A)$ such that, letting $\rho^{\prime}=\min \left(j(A) \backslash\left(\kappa^{\prime}+1\right)\right), \kappa^{\prime}$ is $\left(j(A), \rho^{\prime}+1\right)$-supercompact, is true (as witnessed by $\kappa)$. So the same is true of $A$ in $\mathrm{V}$. So let $\bar{\kappa}$ be a regular limit point of $A$ which witnesses the statement, and let $\bar{\rho}=\min (A \backslash(\bar{\kappa}+1))$. Then in $\mathrm{V}_{\kappa}, \bar{\kappa}$ 
is fully reflecting and $(\bar{A}, \bar{\rho}+1)$-supercompact, where $\bar{A}=A \cap(\rho+1)$ is the set of regular fully reflecting cardinals (in $\mathrm{V}_{\kappa}$ ) less than or equal to $\rho$.

In fact, the argument shows that almost hugeness is strictly stronger than the assumption of the lemma.

I left gaps in the regular cardinals at which the maximality principle is forced below the measurable cardinal $\kappa$, in order to be able to lift the supercompact embedding and to find the derived ultrafilter back in $\mathrm{V}[G]$.

When only interested in weak compactness, I can do without those gaps:

Lemma 3.5. Let $\kappa<\rho$ be regular fully reflecting cardinals. Let $A$ be a set of regular fully reflecting cardinals such that $A \cap \kappa$ is cofinal in $\kappa$ and $\rho=\min (A \backslash$ $(\kappa+1))$. Assume that $\kappa$ is $(A, \rho+1)$-supercompact. Then there is a forcing $\mathbb{P}$ which yields an extension $\mathrm{V}[G]$ such that $\kappa$ is weakly compact in $\mathrm{V}[G]$ and $\mathrm{MP}_{<\bar{\kappa} \text {-dir. cl. }}\left(H_{\bar{\kappa}^{+}}\right)$holds at every regular $\bar{\kappa} \leq \kappa$.

Proof. Let $\mathbb{P}$ be the canonical collapse induced by $A \cap(\rho+1)$. Let $G$ be $\mathbb{P}$-generic over V. So the maximality principle holds up to $\kappa$ in $\mathrm{V}[G]$, as wished. So it has to be shown that $\kappa$ is weakly compact in $\mathrm{V}[G]$.

Let $j: \mathrm{V} \longrightarrow M$ be an $(A, \rho+1)$-supercompact embedding. We cannot easily lift $j$ to an embedding $j^{\prime}: \mathrm{V}[G] \longrightarrow M[G][H]$, for some $H$ generic for the tail forcing as in the proof of Lemma 3.3. For using the terminology of that proof, $D$ would be a directed set of size $\rho$, and the tail forcing would only be $<\rho$-closed in $M[G]$, since there are no gaps in the iteration. So the master condition argument would fail.

So I will not be able to lift the embedding all the way to $\mathrm{V}[G]$ but only to intermediate forcing extensions which will contain all I need. The intermediate forcing will have a gap, as did the forcing from Lemma 3.3.

Namely, to see that $\kappa$ is weakly compact in $\mathrm{V}[G]$, let $N \in \mathrm{V}[G]$ be a transitive structure with $\kappa \in N$, which is of size $\kappa$ in $\mathrm{V}[G]$. I have to find a weakly compact embedding of $N$ in $\mathrm{V}[G]$. Pick $\bar{\rho}$ such that if $\bar{G}=G \uparrow\left(\mathbb{P}_{\kappa} * \operatorname{Col}(\kappa,<\bar{\rho})\right), N \in \mathrm{V}[\bar{G}]$. This is possible since $\mathbb{P}$ is $\rho$-c.c. Let $\mathbb{P}^{\prime}=\mathbb{P}_{\kappa} * \dot{\operatorname{Col}}(\kappa,<\bar{\rho})$. Since $j$ moves $A$ correctly up to $\rho$, it follows that

$$
j\left(\mathbb{P}^{\prime}\right)=\mathbb{P} * \underbrace{\operatorname{Col}(\tilde{T},<\tilde{R})^{M}}_{\mathscr{\mathbb { Q }}},
$$

where $\min (\tilde{T})=\rho$. Let $\mathbb{Q}:=\dot{\mathbb{Q}}^{G}$, and let $\overline{\mathbb{P}}=\mathbb{P}_{\kappa}$, the canonical collapse induced by $A \cap \kappa$. This time, let

$$
D=\left\{\tau^{G} \mid \exists p \in \bar{G} \exists p^{\prime} \quad j(p)=\left\langle p^{\prime}, \tau\right\rangle \in \mathbb{P} * \dot{\mathbb{Q}}\right\} .
$$

(1) $D \in M[G]$, and $\overline{\bar{D}}^{M[G]}<\rho$. 
(2) $M[G] \models " \mathbb{Q}$ is $<\rho$-directed-closed".

(3) $D$ is a directed subset of $\mathbb{Q}$.

Proof. Let $p, q \in D$. Let $\bar{p}, \bar{q} \in \bar{G}$ be such that, letting $j(\bar{p})=\left\langle p^{\prime}, \dot{p}\right\rangle \in \mathbb{P} * \dot{\mathbb{Q}}$ and $j(\bar{q})=\left\langle q^{\prime}, \dot{q}\right\rangle \in \mathbb{P} * \dot{\mathbb{Q}}, p=\dot{p}^{G}$ and $q=\dot{q}^{G}$. Let $\bar{r} \leq_{\mathbb{P}^{\prime}} \bar{p}, \bar{q}$ with $\bar{r} \in \bar{G}$. So

$(*) j(\bar{r}) \leq_{j\left(\mathbb{P}^{\prime}\right)} j(\bar{p}), j(\bar{q})$.

Viewing $\bar{r}$ as a function with domain $\kappa+1$, it follows that $j(\bar{r} \uparrow \kappa) \uparrow(\kappa+1) \in G$ : Since $\overline{\mathbb{P}}$ is a direct limit,

$$
j(\bar{r} \uparrow \kappa) \uparrow \kappa=\bar{r} \uparrow \kappa \text { and } j(\bar{r} \uparrow \kappa) \uparrow[\kappa, j(\kappa)) \equiv 1 .
$$

So $j(\bar{r})(\kappa)$ is a $\overline{\mathbb{P}}$-name for the empty condition in $\operatorname{Col}(\kappa,<\rho)$ but also in $\operatorname{Col}(\kappa,<\bar{\rho})$. This means that that $j(\bar{r}) \uparrow(\kappa+1) \geq \bar{r} \in \bar{G}$, which shows that $j(\bar{r} \uparrow \kappa) \uparrow(\kappa+1) \in$ $\bar{G}$, as claimed.

Now view $j(\bar{r})$ as a pair $\left\langle r^{\prime}, \dot{r}\right\rangle \in \mathbb{P} * \dot{\mathbb{Q}}$. So $r^{\prime}=j(\bar{r}) \uparrow(\kappa+1) \in \bar{G}$, which means that $r:=\dot{r}^{G} \in D$. By $(*)$,

$$
r^{\prime} \leq_{\mathbb{P}} p^{\prime}, q^{\prime} \text { and } r^{\prime} \Vdash_{\mathbb{P}} \dot{r} \leq_{\mathbb{Q}} \dot{p}, \dot{q} .
$$

Moreover, $r^{\prime} \in \bar{G} \subseteq G$, so $r \leq_{\mathbb{Q}} p, q$, which shows that $D$ is directed.

Now pick a master condition $a \in \mathbb{Q}$ below each condition in $D$. Let $H$ be $\mathbb{Q}$-generic over $\mathrm{V}[G]$ with $a \in H$.

(4) $j$ " $\bar{G} \subseteq G * H$.

Proof of (4). Let $\bar{p} \in \bar{G}$, and view its image as $j(\bar{p})=\left\langle p^{\prime}, \dot{p}\right\rangle \in \mathbb{P} * \dot{\mathbb{Q}}$. Then $p:=\dot{p}^{G} \in D$, and so, $a \leq_{\mathbb{Q}} p$. So $p \in H$. Moreover, the argument in the proof of (3) showed that $p^{\prime} \geq \bar{p}$, so that $p^{\prime} \in \bar{G}$. In particular, $p^{\prime} \in G$. So $j(\bar{p})=\left\langle p^{\prime}, \dot{p}\right\rangle \in G * H$, as claimed.

So by Lemma 2.6, this gives us an elementary embedding

$$
j^{\prime}: \mathrm{V}[\bar{G}] \longrightarrow M[G][H]
$$

which extends $j$. This embedding is definable in $\mathrm{V}[G][H]$. As before, ${ }^{\rho}(M[G]) \cap$ $\mathrm{V}[G] \subseteq M[G]$, because $\mathbb{P}$ has size $\rho$ and $M$ is closed under $\rho$-sequences. Moreover, in $M[G], \mathbb{Q}^{G}$ is $<\rho$-closed, hence so it is in $\mathrm{V}[G]$.

Remember that $N \in \mathrm{V}[\bar{G}]$, so $N \cup\{N\} \subseteq \operatorname{dom}\left(j^{\prime}\right)$. So by setting $\tilde{\jmath}:=j^{\prime} \uparrow N$, we get an elementary embedding

$$
\tilde{\jmath}: N \longrightarrow j^{\prime}(N) \text {. }
$$

Since $N$ has size $\kappa<\rho$ in $\mathrm{V}[G], \tilde{\jmath}$ is an element of $\mathrm{V}[G]$, by the $<\rho$-closedness of $\dot{\mathbb{Q}}^{G}$ in $\mathrm{V}[G]$, and it yields a weakly compact embedding of $N$.

It is worthwhile to note that the forcing notion from the previous lemma is a natural forcing which makes $\kappa$ 's weak compactness indestructible, but it has no closure points. So the situation of [AH01] doesn't apply. 


\subsection{The least weakly compact cardinal can be indestructible}

Lemma 3.5 produces a natural model with a surprising indestructibility property:

Corollary 3.6. In the model of Lemma 3.5, the least weakly compact cardinal is indestructibly weakly compact. In particular, this cardinal is indestructibly weakly compact but not measurable, not even $\Pi_{2}^{1}$-indescribable, and not $\Sigma_{2}$-correct.

Proof. Let $\kappa$ be the least weakly compact cardinal in this model. Since the set of weakly compact cardinals below a measurable cardinal has measure one wrt. every normal measure on that cardinal, $\kappa$ cannot be measurable. It cannot be $\Pi_{2}^{1}$ indescribable, since the $\Pi_{2}^{1}$ statement about $\xi$ that expresses that $\xi$ is weakly compact is true for $\xi=\kappa$ but for no smaller $\zeta$. Finally, the statement "there is a weakly compact cardinal" can be expressed in the form "there is an ordinal $\xi$ and a set $x$ such that $x=\mathrm{V}_{\xi+1}$ and $x$ believes that $\xi$ is regular and every tree of height $\xi$ has a cofinal branch". The statement " $x=\mathrm{V}_{\xi+1}$ " is $\Sigma_{2}$.

This result is reminiscent of the result of Apter and Gitik [AG98] that the least measurable cardinal can be Laver indestructible. In fact, if one is only interested in this corollary, the assumptions can be weakened. I'll show two ways how to do this. The first one works with a partial maximality principle for $<\kappa$-closed forcings, which is of independent interest, and which is still strong enough to draw the conclusion of the previous corollary:

Definition 3.7. Let $\Lambda$ be a collection of formulae, $\Gamma$ a collection of forcing notions, and $P$ be a set. Then $\operatorname{MP}_{\Gamma}^{\Lambda}(P)$ is the principle asserting that every statement $\varphi \in \Lambda$ about parameters in $P$ that's $\Gamma$-forceably necessary is already true.

Let $\operatorname{Col}(\kappa)$ be the class of forcings of the form $\operatorname{Col}(\kappa, \lambda)$ or $\operatorname{Col}(\kappa,<\lambda)$, where $\lambda$ ranges over all ordinals. A closer look at the proof of [Fuc08, Thm. 2.10] yields the following:

Lemma 3.8. Assume that $\kappa<\delta, \kappa$ is regular, $n \geq 1$ and $\mathrm{V}_{\delta}$ is $\Sigma_{n+2}$-correct. Let $P=\mathrm{V}_{\delta}$. Then $\mathrm{MP}_{\mathrm{Col}(\kappa)}^{\Sigma_{n}}(P)$ holds in $\mathrm{V}[G]$, where $G$ is $\mathrm{V}$-generic for $\mathbb{P}=\operatorname{Col}(\kappa,<$ $\delta)$. If in addition, $\delta$ is regular, then in $\mathrm{V}[G], \mathrm{MP}_{\mathrm{Col}(\kappa)}^{\Sigma_{n}}\left(H_{\kappa^{+}}\right)$holds.

Proof. Let's focus on the first part of the lemma. The second part works like the proof in [Fuc08], enhanced by the argument to follow. Assume that in $\mathrm{V}[G], \varphi(a)$ is a $\operatorname{Col}(\kappa)$-forceably necessary $\Sigma_{n}$-statement about $a \in P$. Let $\operatorname{Col}(\kappa,<\zeta)$ force $\varphi(a)$ to be $\mathrm{Col}(\kappa)$-necessary over $\mathrm{V}[G]$, where $\zeta$ may be picked as large as wished. Let $H$ be $\operatorname{Col}(\kappa,<\zeta)$-generic over $\mathrm{V}[G]$, so that $\varphi(a)$ is $\operatorname{Col}(\kappa)$-necessary in $\mathrm{V}[G][H]$. Then in $\mathrm{V}$, it is also true that $\varphi(a)$ is forced to be $\operatorname{Col}(\kappa)$-necessary by $\operatorname{Col}(\kappa,<\zeta)$, because there is a $\operatorname{Col}(\kappa,<\zeta)$-generic filter over $\mathrm{V}$ such that $\mathrm{V}[G][H]=\mathrm{V}\left[H^{\prime}\right]$. 
So by homogeneity of $\operatorname{Col}(\kappa,<\zeta)$, $\mathbb{1}$ forces via $\operatorname{Col}(\kappa,<\zeta)$ over $\mathrm{V}$ that $\varphi(a)$ is $\operatorname{Col}(\kappa)$-necessary.

The statement that $\operatorname{Col}(\kappa,<\zeta)$ forces that $\varphi(a)$ is $\operatorname{Col}(\kappa)$-necessary is equivalent to saying that for every $\zeta^{\prime} \geq \zeta, \operatorname{Col}\left(\kappa, \zeta^{\prime}\right)$ forces that $\varphi(a)$ is true. This latter statement says: "For every $\zeta^{\prime} \geq \zeta$ and for every $\mathbb{P}, \mathbb{P} \neq \operatorname{Col}\left(\kappa, \zeta^{\prime}\right)$, or $\mathbb{P} \Vdash \varphi(a)$ ". And " $\mathbb{P}=\operatorname{Col}\left(\kappa, \zeta^{\prime}\right)$ " says: For all $x \in \mathbb{P}, x$ is a function from an ordinal less than $\kappa$ to $\zeta^{\prime}$, and for all functions $y$ from an ordinal less than $\kappa$ to $\zeta^{\prime}, y \in \mathbb{P}$. This is a $\Pi_{1}$ statement. And " $\mathbb{P} \Vdash \varphi(a)$ " is a $\Sigma_{n}$ statement in the parameters $\mathbb{P}$ and $a$. So altogether, we get that the statement that $\operatorname{Col}(\kappa,<\zeta)$ forces $\varphi(a)$ to be $\operatorname{Col}(\kappa)$-necessary is a $\Pi_{n+1}$ statement, and hence, the statement that there is such a $\zeta$ is $\Sigma_{n+2}$.

So since $\mathrm{V}_{\delta} \prec_{\Sigma_{n+2}} \mathrm{~V}$, it is true in $\mathrm{V}_{\delta}$ that $\varphi(a)$ is $\operatorname{Col}(\kappa)$-forceably necessary. Let $\bar{\zeta}<\delta$ be such that in $\mathrm{V}_{\delta}, \operatorname{Col}(\kappa,<\bar{\zeta})$ forces $\varphi(a)$ to be $\operatorname{Col}(\kappa)$-necessary. Again, $\bar{\zeta}$ may be picked as large as wished (below $\delta$ ). Using elementarity upwards this time, it follows that in $\mathrm{V}, \operatorname{Col}(\kappa,<\bar{\zeta})$ forces $\varphi(a)$ to be necessary. By [Fuc08, Cor. 2.4], $\operatorname{Col}(\kappa,<\bar{\zeta}) \times \operatorname{Col}(\kappa,<\delta)$ is forcing equivalent to $\operatorname{Col}(\kappa,<\delta)$. This is because by the reflection properties of $\delta$, for every $\mu<\delta, \mu^{<\kappa}<\delta$. It follows that $\mathrm{V}[G]$ can be viewed as $\mathrm{V}[\tilde{G}][H]$, where $\tilde{G}$ is $\operatorname{Col}(\kappa,<\bar{\zeta})$-generic over $\mathrm{V}$, and $H$ is $\operatorname{Col}(\kappa,<\delta)$-generic over $\mathrm{V}[\tilde{G}]$. Note that the definitions of all these forcings are absolute between all the involved models. $\varphi(a)$ is $\operatorname{Col}(\kappa)$-necessary in $\mathrm{V}[\tilde{G}]$, and so, it is true in $\mathrm{V}[\tilde{G}][H]=\mathrm{V}[G]$. This was to be shown.

By methods introduced in [Fuc08], it follows that

$$
\mathrm{MP}_{\mathrm{Col}(\kappa)}^{\Lambda}(P) \Longrightarrow \mathrm{MP}_{<\kappa \text {-directed-closed }}^{\Lambda}(P) \Longrightarrow \mathrm{MP}_{<\kappa \text {-closed }}^{\Lambda}(P)
$$

Many other facts about the full Maximality Principles go through for the partial ones as well, like, e.g., that the partial Maximality Principle for closed forcings at $\kappa$ is preserved under $<\kappa^{+}$-closed forcing, and the one for directed closed forcings is preserved by $<\kappa^{+}$-directed-closed forcing.

So by adapting the construction of canonical collapses to iterated collapses of the form $\operatorname{Col}(\mu,<\delta)$, where $\delta$ doesn't necessarily have to be regular, the following Lemma can be proved by changing the proof of Lemma 3.5 in the obvious way.

Lemma 3.9. Let $\kappa<\rho$. Let $A$ be a set of $\Sigma_{n+2}$-reflecting cardinals such that $A \cap \kappa$ is cofinal in $\kappa$. Let $\rho=\min (A \backslash(\kappa+1))$, and assume that $\kappa$ is $(A, \rho+1)$ supercompact. Then there is a forcing $\mathbb{P}$ which yields an extension $\mathrm{V}[G]$ such that $\kappa$ is weakly compact in $\mathrm{V}[G]$ and $\mathrm{MP}_{<\bar{\kappa} \text {-directed-closed }}^{\Sigma_{n}}\left(H_{\bar{\kappa}} \cup\{\bar{\kappa}\}\right)$ holds at every regular $\bar{\kappa} \leq \kappa$.

Since the statement that $\kappa$ is weakly compact is a $\Sigma_{2}$-statement about $\kappa$, it follows that in the model of the previous lemma, the least weakly compact cardinal is indestructible. In fact, it is possible to weaken the assumptions further, if one 
is only interested in producing a model where the least weakly compact cardinal is indestructible.

Theorem 3.10. If a supercompact cardinal is consistent, then so is the statement that the least weakly compact cardinal is indestructibly weakly compact. More specifically, if there is a supercompact cardinal $\kappa$ and $a \delta>\kappa$ such that $\mathrm{V}_{\delta}=\mathrm{ZFC}$, then there is a forcing notion which forces that a proper initial segment of the universe is a model of the theory ZFC + "the least weakly compact cardinal is indestructible".

Proof. I shall define an iteration $\left\langle\left\langle\mathbb{P}_{\alpha}, \dot{\mathbb{Q}}_{\alpha}\right\rangle \mid \alpha<\zeta\right\rangle$ by recursion on $\alpha$. It will turn out afterwards what the length of the iteration will be less than $\kappa$.

The beginning is as usual: $\mathbb{P}_{0}$ is trivial forcing.

Now suppose $\alpha \leq \kappa$ is a limit ordinal and $\left\langle\left\langle\mathbb{P}_{\beta}, \dot{\mathbb{Q}}_{\beta}\right\rangle \mid \beta<\alpha\right\rangle$ has been defined already. Then $\mathbb{P}_{\alpha}$ is the limit of the previous iteration, with Easton support. So if $\alpha$ is a regular limit cardinal, we take the direct limit, and otherwise, we take the inverse limit.

The interesting case is that $\left\langle\left\langle\mathbb{P}_{\beta}, \dot{\mathbb{Q}}_{\beta}\right\rangle \mid \beta<\alpha\right\rangle$ has been defined already, where $\alpha \leq \kappa$. It is now clear what $\mathbb{P}_{\alpha}$ is, as well. In case $\alpha<\kappa$, we have to specify $\dot{\mathbb{Q}}_{\alpha}$. By construction, for $\beta \leq \alpha$, there will be $\kappa_{\beta}$ and $\lambda_{\beta}$ such that $\dot{\mathbb{Q}}_{\beta}$ is a $\mathbb{P}_{\beta}$-name for $\operatorname{Col}\left(\kappa_{\beta},<\lambda_{\beta}\right)$. It will be the case that if $\bar{\beta}<\beta<\alpha, \kappa_{\bar{\beta}}<\lambda_{\bar{\beta}}<\kappa_{\beta}<\lambda_{\beta}$. The iteration is a weakly homogeneous forcing notion. Let $\kappa_{\beta}$ be the least $\bar{\kappa}$ such that $\mathbb{P}_{\alpha}$ forces that $\bar{\kappa}$ is weakly compact. Inductively, it will be the case that $\kappa_{\alpha} \geq \sup _{\beta<\alpha} \lambda_{\beta}$. Let $\delta_{\alpha}$ be the least $\zeta>\kappa_{\alpha}$ such that $\mathrm{V}_{\zeta}=$ ZFC. Now ask the question:

Does $\mathbb{P}_{\alpha} * \dot{\operatorname{Col}}\left(\kappa_{\alpha},<\nu\right)$ force that $\kappa_{\alpha}$ is weakly compact, for every $\nu<\delta_{\alpha}$ ?

If so, then $\mathrm{V}_{\delta_{\alpha}}^{\mathbb{P}_{\alpha}}$ is a model of ZFC which thinks that $\kappa_{\alpha}$ is the least weakly compact cardinal, and it is indestructible.

If, on the other hand, the answer is no, then let $\lambda_{\alpha}$ be least such that $\mathbb{P}_{\alpha} *$ $\operatorname{Col}\left(\kappa_{\alpha},<\lambda_{\alpha}\right)$ forces that $\kappa_{\alpha}$ is not weakly compact. So $\lambda_{\alpha}<\delta_{\alpha}$. Let $\dot{\mathbb{Q}}_{\alpha}$ be a $\mathbb{P}_{\alpha}$-name for $\operatorname{Col}\left(\kappa_{\alpha},<\lambda_{\alpha}\right)$.

I claim that the iteration breaks down below $\kappa$, i.e., that the question deciding about the successor case is answered affirmatively at some point below $\kappa$. Then the sought after model has been found already, as pointed out before. So assume, towards a contradiction, that the iteration does not break down below $\kappa$. Then, inductively, the following hold:

1. For $\alpha<\beta<\kappa, \kappa_{\alpha}<\lambda_{\alpha}<\kappa_{\beta}<\lambda_{\beta}$,

2. $\mathbb{P}_{\alpha}$ forces that $\kappa_{\alpha}$ is the least weakly compact cardinal in $\mathrm{V}^{\mathbb{P}_{\alpha}}$,

3. $\kappa_{\alpha}$ is not weakly compact in $\mathrm{V}^{\mathbb{P}_{\beta}}$, for $\alpha<\beta \leq \kappa$, 
4. $\lambda_{\alpha}<\delta_{\alpha}<\kappa_{\beta}$, for $\alpha<\beta<\kappa$.

Let $\delta=\delta_{\kappa}$ be least above $\kappa$ such that $\mathrm{V}_{\delta}=$ ZFC, and let $j: \mathrm{V} \longrightarrow M$ witness the $\delta^{<\kappa}$-supercompactness of $\kappa$. Let $\vec{\kappa}=\left\langle\kappa_{\alpha} \mid \alpha<\kappa\right\rangle, \vec{\lambda}=\left\langle\lambda_{\alpha} \mid \alpha<\kappa\right\rangle$ and $\vec{\delta}=\left\langle\delta_{\alpha} \mid \alpha<\kappa\right\rangle$. We get that

$$
j\left(\mathbb{P}_{\kappa}\right)=\mathbb{P}_{\kappa} * \dot{\operatorname{Col}}(\kappa,<j(\vec{\lambda})(\kappa)) * \dot{\mathbb{Q}}
$$

Namely, $j(\mathbb{P}) \uparrow \kappa=\mathbb{P}_{\kappa}$, since $\kappa$ is the critical point of $j$. Further, $\kappa$ is weakly compact in $M^{\mathbb{P}_{\kappa}}$, which can be seen using the argument of the proof of Lemma 2.7. So this shows that $j(\vec{\kappa})_{\kappa}=\kappa$, by item 2. Moreover,

$$
\lambda^{\prime}:=j(\vec{\lambda})_{\kappa}<\delta
$$

This is because $\mathrm{V}_{\delta}=\mathrm{V}_{\delta}^{M}$, so $\delta$ is least above $\kappa$ with $\mathrm{V}_{\delta} \models$ ZFC from the point of view of $M$ as well as of $\mathrm{V}$. So, arguing in $M$, if $\bar{G}$ is $\mathbb{P}_{\kappa}$-generic over $M$, then $\mathrm{V}_{\delta}^{\mathrm{V}[G]}=\mathrm{V}_{\delta}[G] \models$ ZFC, which means that the least $\zeta>\kappa$ such that in $\mathrm{V}[G]$, $\mathrm{V}_{\zeta}=\mathrm{ZFC}$, is at most $\delta$. So $j(\vec{\lambda})_{\kappa}<j(\vec{\delta})_{\kappa} \leq \delta$. Now let

$$
\mathbb{P}=\mathbb{P}_{\kappa} * \dot{\operatorname{Col}}\left(\kappa,<\lambda^{\prime}\right)
$$

Then

$$
j(\mathbb{P})=\mathbb{P} * \dot{\mathbb{Q}}
$$

and there is a gap between $\overline{\overline{\mathbb{P}}}^{M}$ and the closedness of the tail forcing $\dot{\mathbb{Q}}$. So, letting $G$ be $\mathbb{P}$-generic over $\mathrm{V}$, the argument of the proof of 3.3 shows that there is an $H$ which is $\dot{\mathbb{Q}}^{G}$-generic over $\mathrm{V}$, such that $j$ lifts to $j^{\prime}: \mathrm{V}[G] \longrightarrow M[G][H]$. We find the normal ultrafilter on $\kappa$ derived from $j^{\prime}$ already in $\mathrm{V}[G]$, so that $\kappa$ is measurable in $\mathrm{V}[G]$. This is a contradiction, since $\kappa$ is the least weakly compact cardinal in $\mathrm{V}[G]$ !

This theorem may be a bit surprising, because of the following consideration: In the notation of the theorem, we find $\bar{\kappa}<\kappa$ and an inaccessible $\bar{\delta}<\kappa$ such that in $\mathrm{V}_{\bar{\delta}}[G], \bar{\kappa}$ is indestructibly weakly compact. Assume that the forcing is non-trivial (which is harmless, as one may precede the forcing by adding a Cohen real - being small forcing, this destroys the indestructibility any weakly compact cardinal might have had, by [Ham98] so that in fact, the forcing which makes $\bar{\kappa}$ weakly compact must have been an iteration of length $\bar{\kappa}$ ). Anyway, that forcing has closure points, and so, by the main result of [AH01], $\bar{\kappa}$ is supercompact in $\mathrm{V}_{\bar{\delta}}$. This shows that one cannot start in a minimal universe with a supercompact cardinal (i.e., a universe in which there is no ordinal $\alpha$ such that $\mathrm{V}_{\alpha}$ is a model of "ZFC + there is a supercompact cardinal") and carry out the argument; this would produce a contradiction. By the way, the fact that if $\kappa$ and $\delta$ are as in the 
statement of the theorem, then there are $\bar{\kappa}<\bar{\delta}<\kappa$ such that $\mathrm{V}_{\bar{\delta}}=$ ZFC and $\bar{\kappa}$ is supercompact in $\mathrm{V}_{\bar{\delta}}$, can be proved using methods from [Mag74]. Namely, he introduced the following concept:

Definition 3.11 ([Mag74]). $\kappa$ is $\beta$ reducible if $\kappa<\beta$ and there is an $\alpha<\beta$ and an elementary embedding $j:\left\langle\mathrm{V}_{\alpha}, \in\right\rangle \longrightarrow\left\langle\mathrm{V}_{\beta}, \in\right\rangle$ such that $j$ is not the identity and such that if $\gamma$ is the critical point of $j$, then $j(\gamma)=\kappa$.

The relevance of this concept is expressed in:

Theorem 3.12 ([Mag74], Thm. 1). $\kappa$ is supercompact iff it is $\beta$ reducible, for every $\beta>\kappa$.

In fact, his proof shows: If $\kappa$ is $\beth_{\lambda}$-supercompact, then it is $\lambda$-reducible, and the witness $\alpha$ to $\lambda$-reducibility is less than $\kappa$. So, returning to the assumptions

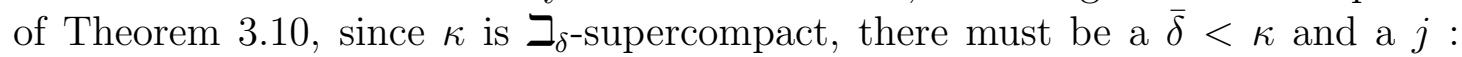
$\left\langle\mathrm{V}_{\bar{\delta}}, \in\right\rangle \longrightarrow\left\langle\mathrm{V}_{\delta}, \in\right\rangle$ with critical point $\bar{\kappa}$, so that $j(\bar{\kappa})=\kappa$. Since $\mathrm{V}_{\delta} \models \mathrm{ZFC}$, the same is true of $\mathrm{V}_{\bar{\delta}}$, and since $j(\bar{\kappa})=\kappa, \bar{\kappa}$ is supercompact in $\mathrm{V}_{\bar{\delta}}$.

So there is no contradiction.

\subsection{Up To Larger Cardinals}

First, let's try to eliminate the gaps between the regular cardinals below a measurable at which the closed Maximality Principle holds. I'll use the following notation: If $\mathbb{P}$ is a forcing iteration, it will usually have the form $\left\langle\mathbb{P}_{\alpha} \mid \alpha<\lambda\right\rangle$, where $\mathbb{P}_{\alpha+1}=\mathbb{P}_{\alpha} * \dot{\mathbb{Q}}_{\alpha}$. Then, for $\alpha<\beta \leq \lambda$, let $\overrightarrow{\dot{Q}} \uparrow[\alpha, \beta)$ be a canonical $\mathbb{P}_{\alpha}$-name for a forcing such that

$$
\mathbb{P}_{\beta} \cong \mathbb{P}_{\alpha} * \overrightarrow{\mathbb{Q}} \uparrow[\alpha, \beta) .
$$

Accordingly, if $G$ is $\mathbb{P}_{\beta}$-generic in this situation, I shall write

$$
G \cong G \uparrow \alpha * G \uparrow[\alpha, \beta)
$$

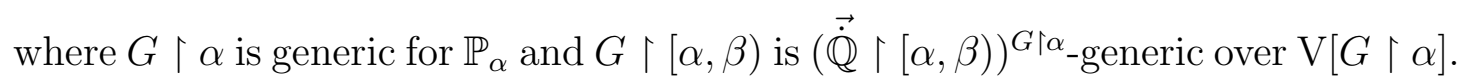

Lemma 3.13. Let $\kappa$ be almost $(A, \rho+1)$-huge, where

$$
\kappa<\rho \in A=\left\{\bar{\rho} \leq \rho \mid \bar{\rho} \text { is regular and } \mathrm{V}_{\bar{\rho}} \prec \mathrm{V}\right\}
$$

and $\rho=\min (A \backslash(\kappa+1))$. Let $j: \mathrm{V} \longrightarrow M$ be almost $(A, \rho+1)$-huge (i.e., $j(A) \cap(\rho+1)=A, j(\kappa)>\rho$ and $\left.{ }^{<j(\kappa)} M \subseteq M\right)$. Let $\mathbb{P}$ be the canonical collapse up to $\rho$, and let $G$ be $j(\mathbb{P})_{j(\kappa)}$-generic over $\mathrm{V}$. Then in $\mathrm{V}[G], \kappa$ is measurable and the directed closed Maximality Principle holds up to $\kappa$. 
Proof. Let $\mathbb{P}=\mathbb{P}_{\kappa+1}$. Since $j$ is almost huge wrt. $A$, it follows that $\mathbb{P}=j(\mathbb{P})_{\kappa+1}$. We shall force with $j(\mathbb{P})_{j(\kappa)}$. So let $G$ be generic for that partial order. Set:

$$
D=\left\{j(p)_{j(\kappa)}^{G} \mid p \in G \uparrow(\kappa+1)\right\},
$$

where I view $p \in \mathbb{P}$, as a sequence $p=\left\langle p_{\alpha} \mid \alpha \leq \kappa\right\rangle$, such that for any $\alpha \leq \kappa$, $p\left\lceil\alpha \in \mathbb{P}_{\alpha}\right.$ and $p\left\lceil\alpha \Vdash_{\mathbb{P}_{\alpha}} p_{\alpha} \in \dot{\mathbb{Q}}_{\alpha}\right.$.

(1) $D \in M[G]$, and $\overline{\bar{D}}^{M[G]}=\rho$.

Proof. The point is that $M[G]$ is $<j(\kappa)$-closed in $\mathrm{V}[G]$, by Lemma 2.5. In particular, $j \uparrow(G \uparrow(\kappa+1))$ as well as $G$ are in $M[G]$, and $D$ is definable from these parameters. Note that $\rho<j(\kappa)$.

(2) $M[G] \models “ j(\overrightarrow{\dot{Q}})_{j(\kappa)}^{G}$ is $<j(\kappa)$-directed-closed.".

Proof. This is because in $M, j(\overrightarrow{\mathbb{Q}})_{j(\kappa)}$ is a $j(\mathbb{P})_{j(\kappa)}$-name for $\operatorname{Col}\left(j(\kappa),<\rho^{\prime}\right)$, where $\rho^{\prime}=j(\rho)>j(\kappa)$.

(3) $D$ is a directed subset of $j(\overrightarrow{\mathbb{Q}})_{j(\kappa)}^{G}$.

Proof. Let $p, q \in D$. Let $\bar{p}, \bar{q} \in G \uparrow(\kappa+1)$ be such that, letting $\dot{p}=j(\bar{p})_{j(\kappa)}$ and $\dot{q}=j(\bar{q})_{j(\kappa)}, p=\dot{p}^{G}$ and $q=\dot{q}^{G}$. Since $G \uparrow(\kappa+1)$ is generic for $\mathbb{P}$, there is an $\bar{r} \leq_{\mathbb{P}} \bar{p}, \bar{q}$ with $\bar{r} \in G \uparrow(\kappa+1)$. So

$(*) j(\bar{r}) \leq_{j(\mathbb{P})} j(\bar{p}), j(\bar{q})$.

I claim that $j(\bar{r}) \uparrow j(\kappa) \in G$. To see this, note that

$$
j(\bar{r}) \uparrow \kappa=\bar{r} \uparrow \kappa \text { and } j(\bar{r}) \uparrow[\kappa, j(\kappa)) \equiv 1 .
$$

So, given any condition $r^{\prime} \in G$ with $\bar{r} \uparrow \kappa=j(\bar{r}) \uparrow \kappa=r^{\prime} \uparrow \kappa$, it follows that $j(\bar{r}) \geq_{j(\mathbb{P})_{j(\kappa)}} r^{\prime}$, which implies that $j(\bar{r}) \uparrow j(\kappa) \in G$. Such an $r^{\prime}$ must exist, of course, since $\bar{r} \in G\lceil\kappa+1$.

Let now $\dot{r}=j(\bar{r})_{j(\kappa)}$ and $r=\dot{r}^{G}$. So $r \in D$. By $(*)$,

$$
j(\bar{r}) \uparrow j(\kappa) \Vdash_{j(\mathbb{P})_{j(\kappa)}} \dot{r} \leq_{j(\overrightarrow{\mathbb{Q}})_{j(\kappa)}} \dot{p}, \dot{q} .
$$

Since $j(\bar{r}) \uparrow j(\kappa) \in G$, this means that $r \leq_{j(\overrightarrow{\mathbb{Q}})_{j(\kappa)}^{G}} p, q$, which shows that $D$ is directed, as claimed.

Now I can pick a master condition $a \in j(\overrightarrow{\dot{Q}})_{j(\kappa)}^{G}$ below each condition in $D$. Let $H$ be $j(\overrightarrow{\mathbb{Q}})_{j(\kappa)}^{G}$-generic over $\mathrm{V}[G]$ with $a \in H$. Define

$$
j^{\prime}: \mathrm{V}[G \uparrow(\kappa+1)] \longrightarrow M[G][H]
$$


by setting $j^{\prime}\left(\tau^{G}\right)=j(\tau)^{G * H}$, for $\tau \in \mathrm{V}^{\mathbb{P}}$. Both the correctness of this definition and the elementarity of $j^{\prime}$ are shown by the following argument: If $\mathrm{V}[G \uparrow(\kappa+1)] \models$ $\varphi\left(\vec{\tau}^{G}\right)$, then there is a condition $p \in G \uparrow(\kappa+1)$ with $p \Vdash_{\mathbb{P}} \varphi(\vec{\tau})$. By elementarity of $j$, this implies that

$$
M \models j(p) \Vdash_{j(\mathbb{P})} \varphi(j(\vec{\tau})) .
$$

Now $j(p)_{j(\kappa)}^{G} \in D$, and so, $j(p)_{j(\kappa)}^{G} \geq_{j(\overrightarrow{\mathbb{Q}})_{j(\kappa)}^{G}} a \in H$. So, in particular, $j(p)_{j(\kappa)}^{G} \in H$. Moreover, in the proof of (3) it was shown that $j(p) \uparrow j(\kappa) \in G$. So $j(p) \in G * H$, which shows that

$$
M[G][H] \models \varphi\left(j(\vec{\tau})^{G * H}\right),
$$

as wished.

Now let $U$ be the normal ultrafilter on $\mathcal{P}(\kappa)$ derived from $j^{\prime}$. $U$ is an element of $\mathrm{V}[G][H]$, but I aim to show that $U \in \mathrm{V}[G]$ already. Note that $j(\kappa)$ is an inaccessible cardinal in $\mathrm{V}[G]$. I have already mentioned that $M[G]$ is closed under $<j(\kappa)$-sequences in $\mathrm{V}[G]$. Since moreover $M[G]$ believes that $j(\overrightarrow{\mathbb{Q}})_{j(\kappa)}^{G}$ is $<j(\kappa)$ closed, this implies that the same is true in $\mathrm{V}[G]$. This will exploited in the following, finally putting the full available closedness to use.

Note that $\left(2^{\kappa}\right)^{\mathrm{V}[G]}<j(\kappa)$. So $U$ is a set of cardinality less than $j(\kappa)$ in $\mathrm{V}[G][H]$, and it is contained in $\mathrm{V}[G]$, by the closedness of the tail forcing. So since in $\mathrm{V}[G]$, $j(\overrightarrow{\mathbb{Q}})_{j(\kappa)}^{G}$ is $<j(\kappa)$-closed, it follows that $U \in \mathrm{V}[G]$. By the same argument, $U$ is a normal ultrafilter in $\mathrm{V}[G]$, since it is $\mathrm{V}[G \uparrow(\kappa+1)]$-normal, but $\mathrm{V}[G]$ has no more subsets of $\kappa$ or regressive functions on $\kappa$ than $\mathrm{V}[G \uparrow(\kappa+1)]$ has, since $j(\overrightarrow{\mathbb{Q}}) \uparrow[\kappa+2, j(\kappa))^{G \uparrow(\kappa+1)}$ is $<\rho$-closed in $\mathrm{V}[G \uparrow(\kappa+1)]$ and $\rho>\kappa$. So $\kappa$ is measurable in $\mathrm{V}[G]$.

The purpose of the following lemma is to collect some aspects relevant to my purposes in which external supercompactness embeddings behave like internal ones.

Lemma 3.14. Let $\mathcal{P}$ and $\mathcal{Q}$ be inner models, $j: \mathcal{P} \longrightarrow \mathcal{Q}$ an elementary embedding with critical point $\kappa<\lambda, \lambda<j(\kappa)$ and ${ }^{\lambda} \mathcal{Q} \subseteq \mathcal{Q}$. Let

$$
\mathcal{F}=\left\{x \in\left(\mathcal{P}_{\kappa} \lambda\right)^{\mathcal{P}} \mid j^{“} \lambda \in j(x)\right\}
$$

be the $\lambda$-supercompactness measure on $\left(\mathcal{P}_{\kappa} \lambda\right)^{\mathcal{P}}$ derived from $j .{ }^{16}$ Then $\mathcal{F}$ is a fine $\mathcal{P}$-normal measure on $\mathcal{P}_{\kappa} \lambda \cap \mathcal{P}$. Moreover, letting $\pi: \mathcal{P} \longrightarrow_{\mathcal{F}} \overline{\mathcal{Q}}$ be the ultrapower of $\mathcal{P}$ by $\mathcal{F}$, there is an elementary embedding $k: \overline{\mathcal{Q}} \longrightarrow \mathcal{Q}$, defined by

$$
k\left([f]_{\mathcal{F}}^{\mathcal{P}}\right)=j(f)\left(j^{“} \lambda\right) .
$$

\footnotetext{
${ }^{16}$ So $\mathcal{F}=\left\{A \in\left(\mathcal{P}_{\kappa} \lambda\right) \cap \mathcal{P} \mid j\right.$ “ $\left.\lambda \in j(A)\right\}$.
} 
Here, $[f]_{\mathcal{F}}^{\mathcal{P}}$ denotes the image of the equivalence class of functions in $\mathcal{P}$ which are equivalent modulo $\mathcal{F}$ to $f$, under the Mostowski collapse.

The critical point of $k$, if it exists, is at least $\lambda$.

Proof. That $\mathcal{F}$ is a fine ultrafilter on $\mathcal{P}_{\kappa}(\lambda)^{\mathcal{P}}$ is straightforward. Let's check its normality: Let $A \in \mathcal{F}$, and let $f: A \longrightarrow \lambda$ be a choice function, and $f \in \mathcal{P}$. We know that $j$ " $\lambda \in j(A)$. So $j(f)\left(j^{\prime \prime} \lambda\right) \in j$ " $\lambda$. In particular, $j(f)(j$ " $\lambda)$ is in the image of $j$, so we can let $\alpha=j^{-1}(j(f)(j$ " $\lambda))<\lambda$. Then $j$ " $\lambda$ is a member of $j(\{x \in A \mid f(x)=\alpha\})$, which means that the set of $x \in A$ with $f(x)=\alpha$ is in $\mathcal{F}$.

The proof that $k$, as defined in the statement of the lemma, is elementary, is standard; the point is that Łoś's theorem stays valid for external ultrapowers. So one can argue that

$$
\begin{aligned}
\overline{\mathcal{Q}} \models \varphi\left([f]_{\mathcal{F}}^{\mathcal{P}}\right) & \Longleftrightarrow\{x \mid \mathcal{P}=\varphi(f(x))\} \in \mathcal{F} \\
& \Longleftrightarrow j “ \lambda \in\{x \mid \mathcal{Q} \models \varphi(j(f)(x))\} \\
& \Longleftrightarrow \mathcal{Q} \models \varphi\left(j(f)\left(j^{\prime \prime} \lambda\right)\right) .
\end{aligned}
$$

A crucial observation which stays true for external ultrapowers is that $[\mathrm{id}]_{\mathcal{F}}^{\mathcal{P}}=$ $\pi$ " $\lambda$ : If $a \in[\mathrm{id}]_{\mathcal{F}}^{\mathcal{P}}$, then $a=[f]_{\mathcal{F}}^{\mathcal{P}}$, for some $f \in \mathcal{P}$. So $\{x \mid f(x) \in x\} \in \mathcal{F}$, which means that $f$ is a choice function on a measure one set. So there is some $\alpha<\lambda$ with $\{x \mid f(x)=\alpha\} \in \mathcal{F}$, which means precisely that $[f]_{\mathcal{F}}^{\mathcal{P}}=\left[\text { const }_{\alpha}\right]_{\mathcal{F}}^{\mathcal{P}}=\pi(\alpha)$. In the other direction, if $\alpha<\lambda$, then claiming that $\pi(\alpha) \in[i d]_{\mathcal{F}}^{\mathcal{P}}$ amounts to saying that $\{x \mid \alpha \in x\} \in \mathcal{F}$, which is the case, since $\mathcal{F}$ is fine.

A consequence of this observation is that $[f]_{\mathcal{F}}^{\mathcal{P}}=\pi(f)\left(\pi^{\text {" }} \lambda\right)$. This can be used to show that any $\alpha<\lambda$ is represented by the function $r_{\alpha}: \mathcal{P}_{\kappa} \lambda^{\mathcal{P}} \longrightarrow \lambda$ defined by

$$
r_{\alpha}(x)=\operatorname{otp}(x \cap \alpha) .
$$

This is because $\left[r_{\alpha}\right]_{\mathcal{F}}^{\mathcal{P}}=\pi\left(r_{\alpha}\right)\left(\pi^{\text {" } \lambda}\right)=\operatorname{otp}\left(\pi^{\text {" } \lambda \cap} \cap(\alpha)\right)=\alpha$.

This, in turn, can be used to show that $k \uparrow \lambda=\mathrm{id}$ : For $\alpha<\lambda, k(\alpha)=$ $k\left(\left[r_{\alpha}\right]_{\mathcal{F}}^{\mathcal{P}}\right)=j\left(r_{\alpha}\right)\left(j^{“} \lambda\right)=\operatorname{otp}\left(j(\alpha) \cap j^{\text {“ }} \lambda\right)=\alpha$, as claimed.

Remark 3.15. A word of caution: If $\mathcal{P}$ is an inner model and $\mathcal{F}$ is a $\mathcal{P}$-normal fine ultrafilter on $\left(\mathcal{P}_{\kappa} \lambda\right)^{\mathcal{P}}$, then it need not be the case that the ultrapower of $\mathcal{P}$ by $\mathcal{F}$, call it $\mathcal{Q}$, is closed under $\lambda$-sequences. Its closure is as follows: If $s=$ $\left\langle\left[f_{i}\right]_{\mathcal{F}} \mid i<\lambda\right\rangle \in{ }^{\lambda} \mathcal{Q}$, and $\left\langle f_{i} \mid i<\lambda\right\rangle \in \mathcal{P}$, then $s \in \mathcal{Q}$. In particular, if ${ }^{\lambda} \mathcal{P} \subseteq \mathcal{P}$, then ${ }^{\lambda} \mathcal{Q} \subseteq \mathcal{Q}$.

Lemma 3.16. Let $\kappa$ be almost huge wrt. $A=\left\{\rho \mid \rho\right.$ is regular and $\left.\mathrm{V}_{\rho} \prec \mathrm{V}\right\}$, where $\kappa \in A \cdot{ }^{17}$ Let $j: \mathrm{V} \longrightarrow M$ be almost huge wrt. $A\left(\right.$ so ${ }^{<j(\kappa)} M \subseteq M$ and

\footnotetext{
${ }^{17}$ This is equivalent to saying that there is an almost huge embedding $j: \mathrm{V} \longrightarrow M$ with critical point $\kappa$, such that $\mathrm{V}_{j(\kappa)} \prec \mathrm{V}$.
} 
$j(A \cap \kappa)=A \cap j(\kappa))$. Then there is a forcing extension of $\mathrm{V}$ in which $\kappa$ is $<j(\kappa)$-supercompact and the directed closed Maximality Principle holds up to $\kappa$.

Proof. Let $\mathbb{P}$ be the canonical collapse below $\kappa$ and let $j: \mathrm{V} \longrightarrow M$ be almost huge wrt. $A$. It follows that $\mathbb{P}=j(\mathbb{P})_{\kappa}$, and moreover, that $j(\mathbb{P})$ is the canonical collapse below $j(\kappa)$. I shall force with $j(\mathbb{P})$. So let $G$ be generic for that partial order.

In order to show that $\kappa$ is supercompact up to $j(\kappa)$ in $\mathrm{V}[G]$, let $\xi<\lambda<j(\kappa)$ be regular limits of $A, \lambda>\xi>\kappa$ (there are unboundedly many such cardinals below $j(\kappa)$ ). In particular, $\xi$ and $\lambda$ are inaccessible, so that $\xi^{\xi}<\lambda$. I shall show that in $\mathrm{V}[G], \kappa$ is $\xi$-supercompact.

Set:

$$
D=\left\{j(p) \uparrow[j(\kappa), j(\lambda))^{G} \mid p \in G \uparrow \lambda\right\} .
$$

To understand the term $(j(p) \uparrow[j(\kappa), j(\lambda)))^{G}$, note that if $p \in j(\mathbb{P})_{\lambda}$, then it is a sequence $p=\left\langle p_{\alpha} \mid \alpha<\lambda\right\rangle$, such that for any $\alpha<\lambda, p \uparrow \alpha \in j(\mathbb{P})_{\alpha}$ and $p\left\lceil\alpha \Vdash_{j(\mathbb{P})_{\alpha}} p_{\alpha} \in j(\overrightarrow{\mathbb{Q}})_{\alpha}\right.$. Let $\dot{\mathbb{P}}_{\text {tail }}=j(j(\overrightarrow{\mathbb{Q}}))\lceil[j(\kappa), j(\lambda))$. Then the term $(j(p) \uparrow$ $[j(\kappa), j(\lambda)))^{G}$ is to be understood in the following way: $j(p)$ can be viewed as a pair $\langle j(p)\lceil j(\kappa), j(p)\lceil[j(\kappa), j(\lambda))\rangle$, where $j(p) \uparrow j(\kappa) \in j(\mathbb{P}), j(p) \uparrow[j(\kappa), j(\lambda))$ is a $j(\mathbb{P})$-name, and $j(p)\left\lceil j(\kappa) \Vdash_{j(\mathbb{P})} j(p) \uparrow[j(\kappa), j(\lambda)) \in \dot{\mathbb{P}}_{\text {tail }}\right.$.

(1) $D \in M[G]$, and $\overline{\bar{D}}^{M[G]}=\lambda$.

Proof. The point is again that $M[G]$ is $<j(\kappa)$-closed in $\mathrm{V}[G]$, by Lemma 2.5. In particular, $j \uparrow(G \uparrow \lambda)$ as well as $G$ are in $M[G]$, and $D$ is definable from these parameters. That $D$ has size $\lambda$ in $M[G]$ is obvious.

(2) $M[G] \models " \dot{\mathbb{P}}_{\text {tail }}^{G}$ is $<j(\kappa)$-directed-closed."

Proof. By elementarity: $\mathbb{1}_{\mathbb{P}}$ forces that $j(\overrightarrow{\mathbb{Q}}) \uparrow[\kappa, \lambda)$ is $<\kappa$-directed-closed. $\quad \square_{(2)}$ Let $\mathbb{P}_{\text {tail }}=\dot{\mathbb{P}}_{\text {tail }}^{G}$.

(3) D is a directed subset of $\mathbb{P}_{\text {tail }}$.

Proof. Let $p, q \in D$. Let $\bar{p}, \bar{q} \in G \uparrow \lambda$ be such that, letting $\dot{p}=j(\bar{p}) \uparrow[j(\kappa), j(\lambda))$ and $\dot{q}=j(\bar{q}) \uparrow[j(\kappa), j(\lambda)), p=\dot{p}^{G}$ and $q=\dot{q}^{G}$. Since $G \uparrow \lambda$ is generic for $j(\mathbb{P})_{\lambda}$, there is an $\bar{r} \leq_{j(\mathbb{P})_{\lambda}} \bar{p}, \bar{q}$ with $\bar{r} \in G \uparrow \lambda$. So

$(*) j(\bar{r}) \leq_{j(j(\mathbb{P}))} j(\bar{p}), j(\bar{q})$. 
Then $j(\bar{r}) \uparrow j(\kappa) \in G$, since $j(\bar{r}) \uparrow \kappa=\bar{r} \uparrow \kappa$ and $j(\bar{r}) \uparrow[\kappa, j(\kappa)) \equiv 1$. So, given any condition $r^{\prime} \in G$ with $j(\bar{r}) \uparrow \kappa=r^{\prime} \uparrow \kappa$, it follows that $j(\bar{r}) \geq_{j(\mathbb{P})\lceil j(\kappa)} r^{\prime}$, which implies that $j(\bar{r})\lceil j(\kappa) \in G$.

Let now $\dot{r}=j(\bar{r}) \uparrow[j(\kappa), j(\lambda))$ and $r=\dot{r}^{G}$. By $(*)$,

$$
j(\bar{r}) \uparrow j(\kappa) \Vdash_{j(\mathbb{P})} \dot{r} \leq_{\dot{\mathbb{P}}_{\text {tail }}} \dot{p}, \dot{q} .
$$

Since $j(\bar{r}) \in G$, this means that $r \leq_{\mathbb{P}_{\text {tail }}} p, q$. Since $r \in D$, this shows that $D$ is directed.

Let $a \in j(j(\overrightarrow{\mathbb{Q}})) \uparrow[j(\kappa), j(\lambda))^{G}$ be a master condition below each condition in

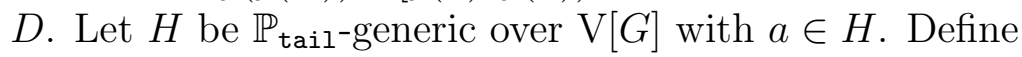

$$
j^{\prime}: \mathrm{V}[G \uparrow \lambda] \longrightarrow M[G][H]
$$

by setting $j^{\prime}\left(\tau^{G \mid \lambda}\right)=j(\tau)^{G * H}$, for $\tau \in \mathrm{V}^{j(\mathbb{P})_{\lambda}}$. To check correctness and elementarity of $j^{\prime}$, suppose $\mathrm{V}[G \uparrow \lambda] \models \varphi\left(\vec{\tau}^{G \uparrow \lambda}\right)$. Then there is a condition $p \in G \uparrow \lambda$ with $p \Vdash_{j(\mathbb{P})_{\lambda}} \varphi(\vec{\tau})$. By elementarity of $j$, this implies that

$$
M \models\left(j(p) \Vdash_{j(j(\mathbb{P}))_{j(\lambda)}} \varphi(j(\vec{\tau}))\right) .
$$

Now $(j(p) \uparrow[j(\kappa), j(\lambda)))^{G} \in D$, and so, $(j(p) \uparrow[j(\kappa), j(\lambda)))^{G} \geq a \in H$, hence $(j(p) \uparrow[j(\kappa), j(\lambda)))^{G} \in H$. Since in the proof of (3) it was shown that $j(p) \uparrow$ $j(\kappa) \in G$, it follows that $j(p) \in G * H$, which shows that

$$
M[G][H] \models \varphi\left(j(\vec{\tau})^{G * H}\right),
$$

as wished.

Note that $j^{\prime}$ is defined in $\mathrm{V}[G][H]$. I want now to pull (part of) it back to $\mathrm{V}[G]$. So let $\mathcal{F}$ be the $\xi$-supercompactness measure on $\mathcal{P}_{\kappa} \xi^{\mathrm{V}[G \mid \lambda]}$ derived from $j^{\prime}$,

$$
\mathcal{F}=\left\{X \subseteq\left(\mathcal{P}_{\kappa} \xi\right)^{\mathrm{V}[G\lceil\lambda]} \mid X \in \mathrm{V}[G \uparrow \lambda] \wedge j^{\prime \prime} \xi \in j^{\prime}(X)\right\} .
$$

(4) $\left(\xi^{<\kappa}\right)^{\mathrm{V}[G \mid \lambda]}=\left(\xi^{<\kappa}\right)^{\mathrm{V}[G]}$.

Proof. The point is that $\xi$ is an inaccessible cardinal in both models, because it is a regular limit of $A$.

(5) $\mathcal{P}\left(\mathcal{P}_{\kappa} \xi\right)^{\mathrm{V}[G \mid \lambda]}=\mathcal{P}\left(\mathcal{P}_{\kappa} \xi\right)^{\mathrm{V}[G]}$

Proof. First, note that

$$
\left(\mathcal{P}_{\kappa} \xi\right)^{\mathrm{V}[G\lceil\lambda]}=\left(\mathcal{P}_{\kappa} \xi\right)^{\mathrm{V}[G]}
$$

This is because the forcing $j(\overrightarrow{\mathbb{Q}}) \uparrow[\lambda, j(\kappa))^{G \uparrow \lambda}$ is $<\lambda$-closed in $\mathrm{V}[G \uparrow \lambda]$. Moreover, $\mathcal{P}_{\kappa} \xi$ has size $\xi$ both in $\mathrm{V}[G]$ and in $\mathrm{V}[G \uparrow \lambda]$, by (4). So, using a bijection between 
$\mathcal{P}_{\kappa} \xi$ and $\xi$ in $\mathrm{V}[G \uparrow \lambda]$ (which then is also a bijection between $\mathcal{P}_{\kappa} \xi$ and $\xi$ in $\mathrm{V}[G]$ ), $\mathcal{P}_{\kappa} \xi$ may be identified with $\xi$, in the sense that it suffices to show that

$$
\mathcal{P}(\xi)^{\mathrm{V}[G\lceil\lambda]}=\mathcal{P}(\xi)^{\mathrm{V}[G]} .
$$

But this is again true by the fact that $j(\overrightarrow{\mathbb{Q}}) \uparrow[\lambda, j(\kappa))^{G \uparrow \lambda}$ is $<\lambda$-closed in $\mathrm{V}[G \uparrow \lambda]$.

(6) $\mathcal{F} \in \mathrm{V}[G]$.

Proof. Working in $\mathrm{V}[G][H]$, the cardinality of $\mathcal{F}$ is the same as the cardinality of $\left(2^{\xi^{<\kappa}}\right)^{\mathrm{V}[G \mid \lambda]}$, which is the same as the cardinality of $\left(2^{\xi}\right)^{\mathrm{V}[G\lceil\lambda]}$. But since $\lambda$ is inaccessible in $\mathrm{V}[G \uparrow \lambda],\left(2^{\xi}\right)^{\mathrm{V}[G \mid \lambda]}<\lambda$, so $\mathcal{F}$ has cardinality less than $j(\kappa)$ in $\mathrm{V}[G][H]$. Now it's crucial that in $\mathrm{V}[G], \mathbb{P}_{\text {tail }}$ is $<j(\kappa)$-closed. This is because by $(2), \mathbb{P}_{\text {tail }}$ is $<j(\kappa)$-closed in $M[G]$, and because $M[G]$ is closed under $<j(\kappa)$ sequences by Lemma 2.5, $\mathbb{P}_{\text {tail }}$ is $<j(\kappa)$-closed in $\mathrm{V}[G]$ as well. Now $\mathcal{F}$ is the range of a sequence of elements of $\mathrm{V}[G \uparrow \lambda]$ which has length $<j(\kappa)$. So the sequence, and hence $\mathcal{F}$, is in $\mathrm{V}[G]$. $\quad \square_{(6)}$

Since $\mathcal{F}$ is derived from a $\xi$-supercompact embedding, it must be $\mathrm{V}[G \uparrow \lambda]$ normal, by Lemma 3.14. But then it is also $\mathrm{V}[G]$-normal, since $\mathrm{V}[G]$ and $\mathrm{V}[G \uparrow \lambda]$ have the same choice functions $f: A \longrightarrow \mathcal{P}_{\kappa} \xi$ with $A \in \mathcal{F}$ and $f(x) \in x$, f.a. $x$ (the proof of (5) shows that they have the same functions with domain $\mathcal{P}_{\kappa} \xi$ and range contained in $\mathrm{V}[G \uparrow \lambda]$ ). So $\mathcal{F}$ is a $\xi$-supercompactness measure on $\mathcal{P}_{\kappa} \xi$ in $\mathrm{V}[G]$.

Remark 3.17. The consistency strength of a huge cardinal is strictly higher than the assumption of Lemma 3.16, namely an almost huge embedding $j: \mathrm{V} \longrightarrow M$ with critical point $\kappa$ such that $\mathrm{V}_{\kappa} \prec \mathrm{V}_{j(\kappa)} \prec \mathrm{V}$.

Before turning to the proof, I need a concrete characterization of almost hugeness that I can work with. The following definition and theorem is from [Kan03, p. 333 f.].

Definition 3.18. Let $\kappa \leq \gamma<\delta$, and let $\mathcal{F}$ be a measure on $\mathcal{P}_{\kappa} \delta$. Then

$$
\mathcal{F} \downarrow \gamma=\{\{x \cap \gamma \mid x \in A\} \mid A \in \mathcal{F}\} .
$$

$\left\langle\mathcal{F}_{\gamma} \mid \kappa \leq \gamma<\lambda\right\rangle$ is a coherent sequence of supercompactness measures if for every $\gamma \in[\kappa, \lambda), \mathcal{F}_{\gamma}$ is a fine normal measure on $\mathcal{P}_{\kappa} \gamma$, and whenever $\kappa \leq \gamma<\delta<\lambda$, then $\mathcal{F}_{\gamma}=\mathcal{F}_{\delta} \downarrow \gamma$.

Theorem 3.19. $\kappa$ is almost huge iff there are an inaccessible $\lambda>\kappa$ and a coherent sequence of supercompactness measures $\left\langle\mathcal{F}_{\gamma} \mid \kappa \leq \gamma<\lambda\right\rangle$ with the following additional property: If $\kappa \leq \gamma<\lambda$ and $\alpha$ is such that, letting $j_{\gamma}: \mathrm{V} \longrightarrow \mathcal{F}_{\gamma} M_{\gamma}$, 
$\gamma \leq \alpha<j_{\gamma}(\kappa)$, then there is a $\delta \in[\gamma, \lambda)$ such that, letting $j_{\delta}: \mathrm{V} \longrightarrow \mathcal{F}_{\delta} M_{\delta}$ and $k_{\gamma, \delta}: M_{\gamma} \longrightarrow M_{\delta}$ be the canonical embedding defined by

$$
k_{\gamma, \delta}\left(j_{\gamma}(f)\left(j_{\gamma} " \gamma\right)\right)=j_{\delta}(f \uparrow \delta)\left(j_{\delta} " \delta\right)
$$

then $k_{\gamma, \delta}(\alpha)=\delta .^{18}$

Let's call a coherent sequence of supercompactness measures as in the theorem an almost hugeness system.

Proof. The argument of the proof of Remark 3.4 works here as well. Let $j$ : $\mathrm{V} \longrightarrow \mathcal{F} M$ be a huge embedding with critical point $\kappa$, and let $A$ be the set of regular cardinals $\rho$ with $\mathrm{V}_{\rho} \prec \mathrm{V}_{\kappa}$, including $\kappa \cdot{ }^{19}$ Let $\overrightarrow{\mathcal{F}}=\left\langle\mathcal{F}_{\gamma} \mid \kappa \leq \gamma<\lambda\right\rangle$ be the almost hugeness system of measures derived from $j$. I.e., $\lambda=j(\kappa)$ and for $\kappa \leq \gamma<\lambda$,

$$
\mathcal{F}_{\gamma}=\left\{X \subseteq \mathcal{P}_{\kappa} \gamma \mid j " \gamma \subseteq X\right\} .
$$

It was shown in the proof of Theorem 3.19 that $\overrightarrow{\mathcal{F}}$ is indeed an almost hugeness system. Note that $\lambda$ is inaccessible, since it is the image of $\kappa$, and hence is inaccessible in $M$, which is closed under $\lambda$-sequences, so that it is also inaccessible in V. Fix $\gamma \in[\kappa, \lambda)$ for a moment. Then $\left(\mathcal{P}_{\kappa} \gamma\right)^{M}=\mathcal{P}_{\kappa} \gamma$. Also, $\mathcal{F}_{\gamma} \subseteq \mathcal{P}_{\kappa} \gamma$ has cardinality less than $\lambda$, so that $\mathcal{F}_{\gamma} \in M$. So since $M$ is closed under $\lambda$-sequences, $\overrightarrow{\mathcal{F}} \in M$. It is now easy to check that $M$ agrees with $\mathrm{V}$ that $\overrightarrow{\mathcal{F}}$ is an almost hugeness system: Each $\mathcal{F}_{\gamma}$ of course is still a normal ultrafilter on $\mathcal{P}_{\kappa} \gamma$, and the sequence is obviously coherent, since this is true in $\mathrm{V}$. To see that the additional conditions are also satisfied, let $j_{\gamma}^{M}: M \longrightarrow N_{\gamma}$ and $k_{\gamma, \delta}^{M}$ be defined as in Theorem 3.19, for $\kappa \leq \gamma<\delta<\lambda$, in $M$. Suppose $\gamma \leq \alpha<j_{\gamma}^{M}(\kappa)$. Let $\alpha=\left[f_{\alpha}\right]_{\mathcal{F}_{\gamma}}^{M}$, i.e., $\alpha=j_{\gamma}^{M}\left(f_{\alpha}\right)\left(j_{\gamma}^{M}\right.$ “ $\left.\gamma\right)$. This means that in $M$,

$$
\left\{x \in \mathcal{P}_{\kappa} \gamma \mid \operatorname{otp}(x) \leq f_{\alpha}(x)<\kappa\right\} \in \mathcal{F}_{\gamma}
$$

So by absoluteness, this is also true in V, and hence, $\gamma \leq\left[f_{\alpha}\right]_{\mathcal{F}_{\gamma}}<j_{\gamma}(\kappa)$. Since $\overrightarrow{\mathcal{F}}$ is an almost hugeness system in $\mathrm{V}$, there is a $\delta \in[\gamma, \lambda)$ such that $k_{\gamma, \delta}\left(\left[f_{\alpha}\right]_{\mathcal{F}_{\gamma}}\right)=\delta$. In other words, this means that

$$
\left\{x \in \mathcal{P}_{\kappa} \delta \mid f_{\alpha}(x \cap \gamma)=\operatorname{otp}(x)\right\} \in \mathcal{F}_{\delta}
$$

But since $\mathrm{V}$ and $M$ agree about $\mathcal{P}_{\kappa} \delta$, this is also true in $M$, showing that $k_{\gamma, \delta}^{M}(\alpha)=$ $\delta$. So $\overrightarrow{\mathcal{F}}$ is an almost hugeness system in $M$.

\footnotetext{
${ }^{18}$ In the definition of $k_{\gamma, \delta}, f$ is a function with domain $\mathcal{P}_{\kappa} \gamma$. Its lift to $\mathcal{P}_{\kappa} \delta$ is the function $f \uparrow \delta: \mathcal{P}_{\kappa} \delta \longrightarrow \mathrm{V}$ defined by: $(f \uparrow \delta)(x)=f(x \cap \gamma)$.

${ }^{19}$ Starting from a huge embedding $l: \mathrm{V} \longrightarrow M^{\prime}$ with critical point $\kappa$, let $\mathcal{F}$ be the normal measure on $\mathcal{P}_{\kappa} \lambda$ derived from $l$, where $\lambda=j(\kappa)$. Then form $j: \mathrm{V} \longrightarrow \mathcal{F} M$. This will be a huge embedding again. $\mathcal{F}$ has the property that $\left\{x \in \mathcal{P}_{\kappa} \lambda \mid \operatorname{otp}(x)=\kappa\right\} \in \mathcal{F}$.
} 
Let $j^{\prime}: M \longrightarrow \overrightarrow{\mathcal{F}} N$ be the embedding induced by the almost hugeness system:

$$
\left\langle N,\left\langle j_{\gamma, \infty} \mid \kappa \leq \gamma<\lambda\right\rangle\right\rangle=\operatorname{dir} \lim \left(\left\langle N_{\gamma} \mid \kappa \leq \gamma<\lambda\right\rangle,\left\langle k_{\gamma, \delta}^{M} \mid \kappa \leq \gamma<\delta<\lambda\right\rangle\right),
$$

where $N$ is transitive, and $j^{\prime}=j_{\kappa, \infty} \circ j_{\kappa}^{M}$, where $j_{\kappa, \infty}: \operatorname{Ult}\left(M, \mathcal{F}_{\kappa}\right) \longrightarrow N$ is the direct limit embedding and $j_{\kappa}^{M}: M \longrightarrow \mathcal{F}_{\kappa} \operatorname{Ult}\left(M, \mathcal{F}_{\kappa}\right)$ is the ultrapower embedding. Then $j^{\prime}$ is, in $M$, an almost huge embedding with critical point $\kappa \in j(A)$. This was shown in the proof of 3.19 , see [Kan03]. Moreover, $j^{\prime}$ moves $j(A)$ correctly up to $j^{\prime}(\kappa)$. To see this, note the following facts, which are not hard to verify:

- $\operatorname{crit}\left(k_{\gamma, \delta}^{M}\right)>\gamma$,

- $\operatorname{crit}\left(j_{\delta, \infty}\right)>\delta$,

- $\operatorname{crit}\left(k_{\gamma, \delta}\right)>\gamma$

- For $x \subseteq \kappa$ and $\gamma \in[\kappa, \lambda)$,

$$
j(x) \cap(\gamma+1)=j_{\gamma}(x) \cap(\gamma+1) \text { and } j^{\prime}(x) \cap(\gamma+1)=j_{\gamma}^{M}(x) \cap(\gamma+1) .
$$

But it's also clear that for $x \subseteq \kappa$ and $\gamma \in[\kappa, \lambda), j_{\gamma}^{M}(x) \cap \gamma=j_{\gamma}(x) \cap \gamma$. Note that $j^{\prime}(\kappa)=j(\kappa)=\lambda$, which follows from the above and the condition turning a coherent sequence of supercompactness measures into an almost hugeness system. Putting these facts together, it follows that

$$
j^{\prime} \uparrow \mathcal{P}(\kappa)=j \uparrow \mathcal{P}(\kappa) .
$$

So since $j$ obviously moves $j(A)$ correctly up to $\lambda$, so does $j^{\prime}$. And since $j(\kappa) \in$ $j(A)$, the same is true of $j^{\prime}(\kappa)$.

So the statement about $j(\kappa)$ and $j(A)$ that there is an almost huge embedding $j^{\prime}$ with critical point $\kappa^{\prime} \in j(\kappa) \cap j(A)$, which moves $j(A)$ correctly up to $j^{\prime}\left(\kappa^{\prime}\right)$, and such that $j^{\prime}\left(\kappa^{\prime}\right) \in j(A)$, is true in $M$, as witnessed by $\kappa$. So the same statement is true of $\kappa$ and $A$ in V. Pick witnesses $\bar{\jmath}$ and $\bar{\kappa}$ in $\mathrm{V}$. Then $\bar{\jmath} \uparrow \mathrm{V}_{\kappa}$ witnesses that $\mathrm{V}_{\kappa}$ is a model of the desired theory.

Of course, the cardinal up to which the directed closed Maximality Principle holds in the model of the previous lemma is also Woodin. But since the lifting techniques don't work so well with cardinals satisfying variants of strongness, it's natural to replace Woodinness with a similar large cardinal notion which bears closer connections to supercompactness than to strongness. The following definition is inspired by [For].

Definition 3.20. A cardinal $\kappa$ is Woodinized supercompact if for every $A \subseteq \mathrm{V}_{\kappa}$, there is a $\bar{\kappa}<\kappa$ which is $A$-supercompact up to $\kappa$. Analogously, $\kappa$ is Woodinized almost huge if for every $A \subseteq \mathrm{V}_{\kappa}$, there is a $\bar{\kappa}<\kappa$ which is almost huge wrt. $A$ up to $\kappa$. I.e., if for every $\lambda<\kappa$, there is an almost huge $j: \mathrm{V} \longrightarrow M$ with critical point $\bar{\kappa}$, which moves $A$ correctly up to $j(\kappa)$ and $j(\kappa)>\lambda$. 
The concept of Woodinized large cardinals is quite general. So, for example, a Woodin cardinal is a Woodinized strong cardinal.

The final aim is to produce a model in which the closed maximality principle holds up to a Woodinized supercompact cardinal. First, let's produce a model where it holds below a Woodinized supercompact, even though formally, this construction should belong in section 2. But with almost no additional work, the construction produces a model where the closed Maximality Principle holds up to a Woodin cardinal, and the methods used in the proof fit better in the present section.

Lemma 3.21. Let $\kappa$ be a fully reflecting Woodinized almost huge cardinal. Then there is a forcing $\mathbb{P}$ such that if $G$ is $\mathbb{P}$-generic over $\mathrm{V}$, then in $\mathrm{V}[G], \kappa$ is Woodinized supercompact, and the directed closed Maximality Principle holds below $\kappa$.

Proof. Let $A=\left\{\rho \mid \mathrm{V}_{\rho} \prec \mathrm{V}_{\kappa} \wedge \rho\right.$ is regular $\}$. Let $\mathbb{P}=\mathbb{P}_{A}$ be the canonical collapse below $\kappa$. I want to show that $\kappa$ is a Woodinized supercompact cardinal in $\mathrm{V}[G]$, where $G$ is $\mathbb{P}$-generic over $\mathrm{V}$.

So let $B=\dot{B}^{G} \in \mathrm{V}[G], B \subseteq \kappa, \dot{B}$ a nice $\mathbb{P}$-name for a subset of $\check{\kappa}$. Let

$$
\dot{B}=\bigcup_{\alpha<\kappa}\{\check{\alpha}\} \times A_{\alpha}
$$

where $A_{\alpha}$ is an antichain in $\mathbb{P}$.

If $\alpha<\kappa$, then I shall say that $\alpha$ is $\dot{B}$-closed if for all $\beta<\alpha$ and all conditions $p=\left\langle p_{\gamma}\right| \gamma\langle\kappa\rangle \in A_{\beta}$, the support of $p$ is contained in $\alpha$. In other words, for all $\gamma \in[\alpha, \kappa), p_{\gamma}=\mathbb{1}$. So essentially, $\alpha$ is $\dot{B}$-closed if $A_{\beta} \subseteq \mathbb{P}_{\alpha}$, for all $\beta<\alpha$. Clearly, the set $C$ of $\dot{B}$-closed ordinals is club in $\kappa$.

Let $\bar{\kappa}<\kappa$ be such that $\bar{\kappa}$ is $A \oplus \dot{B}$-huge up to $\kappa$, where $A \oplus \dot{B}$ is some subset of $\mathrm{V}_{\kappa}$ coding $A$ and $\dot{B}$; for example $(A \times\{0\}) \cup(\dot{B} \times\{1\})$ would work. Note that in particular, $\bar{\kappa}$ is a limit point of $C$ and of $A$, so that $\bar{\kappa}$ is $\dot{B}$-closed and $\mathrm{V}_{\bar{\kappa}} \prec \mathrm{V}_{\kappa}$.

Let $\xi<\lambda$ be regular limits of $A$ and $C$ less than $\kappa$. I want to show that in $\mathrm{V}[G]$, $\bar{\kappa}$ is $\xi$-supercompact wrt. $B$. So let $j: \mathrm{V} \longrightarrow M$ be an $A \oplus \dot{B}$-huge embedding with $j(\bar{\kappa})>\lambda$, and let $\overline{\mathbb{P}}=\mathbb{P}_{\bar{\kappa}}$. It follows that

$$
j(\overline{\mathbb{P}})=\left(\mathbb{P}_{j(A \cap \bar{\kappa})}\right)^{M}=\mathbb{P}_{j(\bar{\kappa})} ;
$$

the latter equality follows from the $<j(\bar{\kappa})$-closedness of $M$ and from the fact that $j$ moves $A$ correctly up to $j(\bar{\kappa})$.

Note that since $\bar{\kappa} \in A$ and $A$ is an initial segment of the class of all fully reflecting regular cardinals, the assumptions of Lemma 3.16 are satisfied by $\bar{\kappa}$. It was shown in the proof of that lemma (right after claim (3)) that $j$ lifts to

$$
j^{\prime}: \mathrm{V}[G \uparrow \lambda] \longrightarrow M[G \uparrow j(\bar{\kappa})][H],
$$


for some $H$ which is generic over $M[G \uparrow j(\bar{\kappa})]$ for the partial order $\mathbb{P}_{\text {tail }}=(j(j(\overrightarrow{\dot{\mathbb{Q}}} \uparrow$ $\bar{\kappa})) \uparrow[j(\bar{\kappa}), j(\lambda)))^{G\lceil j(\bar{\kappa})} \cdot j^{\prime}$ is defined by:

$$
j^{\prime}\left(\tau^{G \uparrow \lambda}\right)=j(\tau)^{(G\lceil j(\bar{\kappa})) * H} .
$$

I now want to show that $j^{\prime}$ moves $B$ correctly up to $\lambda$. To this end, let $\dot{B} \sqcap \gamma=$ $\bigcup_{\alpha<\gamma}\{\alpha\} \times A_{\alpha}$, and note:

1. If $\alpha \in C$, then $B \cap \alpha=(\dot{B} \sqcap \alpha)^{G\lceil\alpha}$.

2. $\lambda$ is $j(\dot{B})$-closed.

The second point holds since $\lambda$ is a limit of $C$ and $j(C) \cap \lambda=C \cap \lambda$, as $C$ is defined from $\dot{B}$, which is moved correctly by $j$ up to $j(\kappa)>\lambda$. It follows that:

$$
\begin{aligned}
j^{\prime}(B) \cap \lambda & =j^{\prime}(B \cap \bar{\kappa}) \cap \lambda \\
& =j^{\prime}\left((\dot{B} \sqcap \bar{\kappa})^{G \uparrow \bar{\kappa}}\right) \cap \lambda \\
& =j^{\prime}\left((\dot{B} \sqcap \bar{\kappa})^{G \uparrow \lambda}\right) \cap \lambda \\
& =(j(\dot{B} \sqcap \bar{\kappa}))^{G * H} \cap \lambda \\
& =(j(\dot{B} \sqcap \bar{\kappa}) \sqcap \lambda)^{G * H} \\
& =(j(\dot{B}) \sqcap \lambda)^{G * H} \\
& =\left(j\left(\dot{B} \cap \mathrm{V}_{\lambda}\right)\right)^{G * H} \\
& =\left(\dot{B} \cap \mathrm{V}_{\lambda}\right)^{G * H} \\
& =(\dot{B} \sqcap \lambda)^{G * H} \\
& =(\dot{B} \sqcap \lambda)^{G \uparrow \lambda} \\
& =B \cap \lambda .
\end{aligned}
$$

Now let $\mathcal{F}$ be the $\xi$-supercompactness measure on $\left(\mathcal{P}_{\bar{\kappa}} \xi\right)^{\mathrm{V}[G \mid \lambda]}$ derived from $j^{\prime}$ (in $\mathrm{V}[G\lceil j(\bar{\kappa})][H]$ ). Then as in the proof of Lemma 3.16, it follows that actually $\mathcal{F} \in \mathrm{V}\left[G\lceil j(\bar{\kappa})]\right.$, and that $\mathcal{F}$ is a $\xi$-supercompactness measure on $\left(\mathcal{P}_{\bar{\kappa}} \xi\right)^{\mathrm{V}[G\lceil j(\bar{\kappa})]}$.

Since $\overrightarrow{\mathbb{Q}} \uparrow[j(\bar{\kappa}), \kappa)^{G \uparrow j(\bar{\kappa})}$ is $<j(\bar{\kappa})$-closed in $\mathrm{V}[G \uparrow j(\bar{\kappa})]$, and since $j(\bar{\kappa})$ is inaccessible there, it follows that $\mathcal{F}$ is a fine normal ultrafilter on $\mathcal{P}_{\bar{\kappa}} \xi$ from $\mathrm{V}[G]$ 's point of view as well.

Now form

$$
\pi: \mathrm{V}[G] \longrightarrow \mathcal{F} N
$$

Then $\pi$ is (in $\mathrm{V}[G]$ ) a $\xi$-supercompact embedding with critical point $\bar{\kappa}$. It has to be verified that it moves $B$ correctly up to $\xi$.

To this end, Lemma 3.14 is applicable: Let $\bar{\pi}: \mathrm{V}[G \uparrow \lambda] \longrightarrow_{\mathcal{F}} \bar{N}$ be the ultrapower embedding of $\mathrm{V}[G \uparrow \lambda]$ by $\mathcal{F}$, and let $k: \bar{N} \longrightarrow M[G \uparrow j(\bar{\kappa})][H]$ be 
the factor map. By the lemma, $k$ is the identity up to $\xi$. The situation looks as follows:
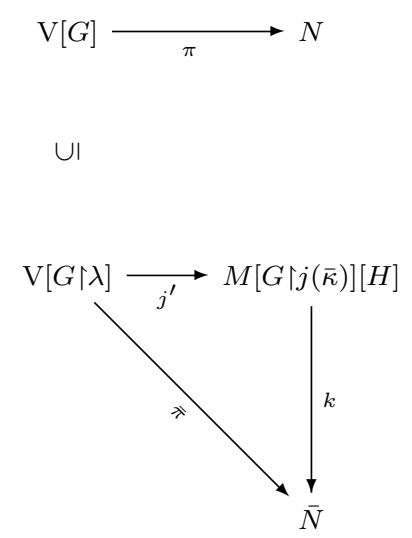

Now let $x \in \mathcal{P}(\bar{\kappa})^{\mathrm{V}[G]}$, and let $\alpha<\xi$. Then

$$
\begin{aligned}
\alpha \in \pi(x) & \Longleftrightarrow\left\{z \in\left(\mathcal{P}_{\bar{\kappa}} \xi\right)^{\mathrm{V}[G]} \mid \operatorname{otp}(z \cap \alpha) \in x\right\} \in \mathcal{F} \\
& \Longleftrightarrow\left\{z \in\left(\mathcal{P}_{\bar{k}} \xi\right)^{\mathrm{V}[G\lceil\lambda]} \mid \operatorname{otp}(z \cap \alpha) \in x\right\} \in \mathcal{F} \\
& \Longleftrightarrow \alpha \in \bar{\pi}(x) \\
& \Longleftrightarrow \alpha=k(\alpha) \in k(\bar{\pi}(x))=j^{\prime}(x) .
\end{aligned}
$$

This argument just used that $\mathrm{V}[G]$ is a forcing extension of $\mathrm{V}[G \uparrow \lambda]$ by a forcing that's $<\bar{\kappa}$-closed - much more is true, of course. In particular, since $j^{\prime}$ moved $B$ correctly up to $\xi$, so does $\pi$.

Corollary 3.22. Let $\kappa$ be a fully reflecting Woodinized almost huge cardinal with a fully reflecting regular cardinal $\rho$ above. Then there is a forcing $\mathbb{P}$ such that if $G$ is $\mathbb{P}$-generic over $\mathrm{V}$, then in $\mathrm{V}[G], \kappa$ is a Woodinized supercompact cardinal and the directed closed Maximality Principle holds up to $\kappa$.

Proof. First force with the forcing of the previous lemma. In the extension, it is still the case that $\mathrm{V}_{\rho} \prec \mathrm{V}$, so further forcing with $\operatorname{Col}(\kappa,<\rho)$ yields a model in which the desired maximality principles hold (the ones down low remain true), and $\kappa$ 's Woodinized supercompactness is preserved as well, because this is a $\Pi_{1}^{1}\left(H_{\kappa}\right)$ property.

I'll close the paper with the following question:

Question 3.23. Is it consistent that the closed Maximality Principle holds up to a supercompact cardinal?

This is a subtle question since it would seem that one has to carry out a proper class canonical collapse in order to produce such a model, while at the same time 
preserving the Maximality Principles, which is very problematic: In general, these principles are not preserved in such an iteration, as one could otherwise force the closed Maximality Principle to hold up to $\infty$, which is impossible, as pointed out in the Introduction - see [Fuc08].

\section{References}

[AG98] Arthur Apter and Moti Gitik. The least measurable can be strongly compact and indestructible. Journal of Symbolic Logic, 63(4):1404-1412, Dec. 1998.

[AH01] Arthur W. Apter and Joel David Hamkins. Indestructible weakly compact cardinals and the necessity of supercompactness for certain proof schemata. Mathematical Logic Quarterly, 47(4):563-571, 2001.

[For] Matthew Foreman. Handbook of Set Theory, chapter Ideals and Generic Elementary Embeddings. Springer.

[Fuc] Gunter Fuchs. Generic embeddings associated to an indestructibly weakly compact cardinal. pages $1-20$. In preparation.

[Fuc08] Gunter Fuchs. Maximality principles for closed forcings: Implications, separations and combinations. Journal of Symbolic Logic, 73(01):276308, March 2008.

[Ham98] Joel David Hamkins. Small forcing makes any cardinal superdestructible. Journal of Symbolic Logic, 63(1):51-58, 1998.

[Ham03] Joel David Hamkins. A simple maximality principle. Journal of Symbolic Logic, 68(2):527-550, June 2003.

[Jec03] Thomas Jech. Set Theory. The Third Millenium Edition. Springer, Berlin, 2003.

[Jen66] Ronald B. Jensen. Independence of the axiom of dependent choices from the countable axiom of choice. Journal of Symbolic Logic, 31:294, 1966.

[JSSS07] Ronald B. Jensen, Ernest Schimmerling, Ralf Schindler, and John R. Steel. Stacking mice. 2007. In preparation.

[Kan03] Akihiro Kanamori. The Higher Infinite. Springer Monographs in Mathematics. Springer, 2 edition, 2003. 
[Lei04] George Leibman. Consistency Strengths of Modified Maximality Principles. PhD thesis, The City University of New York, 2004.

[Mag74] Menachem Magidor. Combinatorial characterization of supercompact cardinals. Proceedings of the American Mathematical Society, 42(1):279285, Jan. 1974.

[Men74] Telis K. Menas. On strong compactness and supercompactness. Annals of Mathematical Logic, 7:327-359, 1974.

[Ste96] John R. Steel. The Core Model Iterability Problem. Lecture Notes in Logic 8. Springer, Berlin, 1996.

[SV01] Jonathan Stavi and Jouko Väänänen. Reflection principles for the continuum. Logic and Algebra, AMS Contemporary Mathematics Series, 302, 2001. 